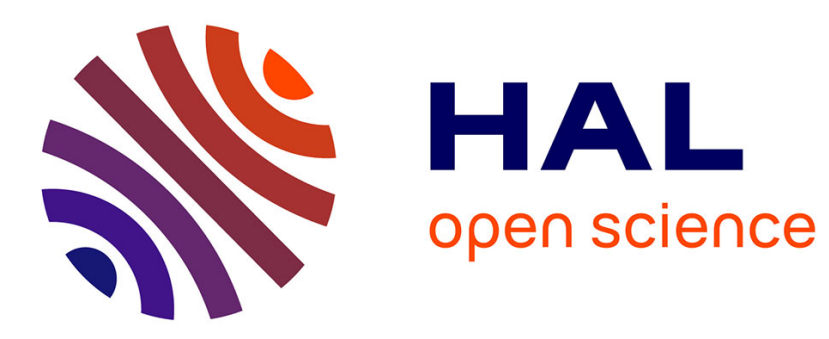

\title{
The minimal seed of turbulent transition in the boundary layer
}

Stefania Cherubini, Pietro de Palma, Jean-Christophe Robinet, Alessandro Bottaro

\section{- To cite this version:}

Stefania Cherubini, Pietro de Palma, Jean-Christophe Robinet, Alessandro Bottaro. The minimal seed of turbulent transition in the boundary layer. Journal of Fluid Mechanics, 2011, pp.221-253. 10.1017/jfm.2011.412 . hal-00782191

\section{HAL Id: hal-00782191 https://hal.science/hal-00782191}

Submitted on 29 Jan 2013

HAL is a multi-disciplinary open access archive for the deposit and dissemination of scientific research documents, whether they are published or not. The documents may come from teaching and research institutions in France or abroad, or from public or private research centers.
L'archive ouverte pluridisciplinaire HAL, est destinée au dépôt et à la diffusion de documents scientifiques de niveau recherche, publiés ou non, émanant des établissements d'enseignement et de recherche français ou étrangers, des laboratoires publics ou privés. 


\title{
The minimal seed of turbulent transition in the boundary layer
}

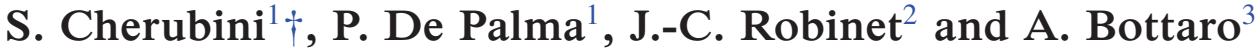 \\ ${ }^{1}$ DIMeG, CEMeC, Politecnico di Bari, Via Re David 200, 70125 Bari, Italy \\ 2 DynFluid Laboratory, Arts et Metiers ParisTech, 151 Boulevard de l'Hopital, 75013 Paris, France \\ ${ }^{3}$ DICAT, Università di Genova, Via Montallegro 1, 16145 Genova, Italy
}

(Received 8 April 2011; revised 20 September 2011; accepted 21 September 2011; first published online 15 November 2011)

This paper describes a scenario of transition from laminar to turbulent flow in a spatially developing boundary layer over a flat plate. The base flow is the Blasius non-parallel flow solution; it is perturbed by optimal disturbances yielding the largest energy growth over a short time interval. Such perturbations are computed by a nonlinear global optimization approach based on a Lagrange multiplier technique. The results show that nonlinear optimal perturbations are characterized by a localized basic building block, called the minimal seed, defined as the smallest flow structure which maximizes the energy growth over short times. It is formed by vortices inclined in the streamwise direction surrounding a region of intense streamwise disturbance velocity. Such a basic structure appears to be a robust feature of the base flow since it is practically invariant with respect to the initial energy of the perturbation, the target time, the Reynolds number and the dimensions of the computational domain. The minimal seed grows very rapidly in time while spreading, and it triggers nonlinear effects which bring the flow to turbulence in a very efficient manner, through the formation of a turbulence spot. This evolution of the initial optimal disturbance has been studied in detail by direct numerical simulations. Using a perturbative formulation of the Navier-Stokes equations, each linear and nonlinear convective term of the equations has been analysed. The results show the fundamental role of the streamwise inclination of the vortices in the process. The nonlinear coupling of the finite amplitude disturbances is crucial to sustain such streamwise inclination, as well as to generate dislocations within the flow structures, and local inflectional velocity distributions. The analysis provides a picture of the transition process characterized by a sequence of structures appearing successively in the flow, namely, $\Lambda$ vortices, hairpin vortices and streamwise streaks. Finally, a disturbance regeneration cycle is conceived, initiated by the fast nonlinear amplification of the minimal seed, providing a possible scenario for the continuous regeneration of the same fundamental flow structures at smaller space and time scales.

Key words: boundary layer stability, nonlinear instability, transition to turbulence 


\section{Introduction}

Despite many efforts in the last century and some breakthroughs, the very nature of transition to turbulence continues to elude the fluid mechanics community, an indication of how formidable and fascinating the process is. It is now clear that several routes to turbulence may exist in a given flow, with different degrees of efficiency, which can be measured in terms of the space and/or time needed to reach the final chaotic state. The original view, consisting of the linear amplification of two-dimensional Tollmien-Schlichting or Rayleigh waves in boundary and shear layers, followed by secondary instability and a nonlinear mixing process capable to redistribute energy among different modes, has been all but replaced by new scenarios focusing on receptivity (Saric, Reed \& Kerschen 2002), transient growth (Chomaz 2005), as well as coherent flow structures (Adrian 2007) and their nonlinear interactions (Eckhardt et al. 2007).

It has been established that in the subcritical regime, when all eigenmodes are damped, disturbances may be amplified by a non-normal growth mechanism arising from the constructive interference of nearly antiparallel eigenfunctions (Schmid \& Henningson 2001). At small initial disturbance levels this growth is linear; to identify the maximum possible growth, the concept of optimal perturbations was introduced. Optimal disturbances are defined as those initial flow states which yield the largest amplification of the disturbance energy density over a time/space interval (Farrell 1988; Luchini 2000). For the case of the boundary layer at low Reynolds number, of interest here, the result, obtained for a laminar profile in a local setting, is that linear optimal perturbations consist of pairs of counter-rotating streamwise vortices, capable of eliciting streamwise streaks by the lift-up effect (Landahl 1980). If growth is sufficient, such elongated structures can experience secondary instability and breakdown (Schoppa \& Hussain 2002; Brandt, Schlatter \& Henningson 2004). For sufficiently high values of the Reynolds number and of the initial perturbation amplitude, nonlinear effects may set in and trigger bypass transition, by generating a turbulent spot which rapidly amplifies and spreads, leading the flow towards the fully turbulent state.

A common objection to this transition scenario is that optimal streamwise-invariant initial disturbances can be rarely observed in a real non-parallel boundary-layer flow. In fact, in most practical cases, the flow undergoes transition by receptively selecting and amplifying free stream turbulence perturbations (Jacobs \& Durbin 2001; Brandt et al. 2004), or localized disturbances, such as those arising from the presence of roughness elements or gaps on the wall. Therefore, it is important to justify the choice of employing an initial optimal perturbation for studying the route to transition. As already discussed by Luchini (2000), optimal perturbations can be used to unravel the most efficient amplification mechanisms which dominate the growth of the perturbation over short time/space scales. In a linear framework, this happens when the first and the second singular values of the evolution operator are well separated, such as in the case of the boundary layer (Luchini 2000). Therefore, perturbations having a large projection onto the optimal one would provide a large contribution to the energy amplification. This could lead to a flow dominated by optimal and near-optimal streaks even when the flow is excited by free stream turbulence, as suggested by the comparison of the optimization results obtained by Luchini (2000) with the experimental data of Westin et al. (1994). However, many studies have by now demonstrated that linear optimals obtained by a local approach are inefficient at triggering transition and, for example, the so-called oblique transition mechanism 
succeeds in triggering transition at a much lower initial disturbance level than linear optimals (see, for instance, Reddy et al. 1998).

Following these ideas, Cherubini et al. (2010b) attempted to identify, in a linear framework, initial localized disturbances capable of provoking breakdown to turbulence most effectively in a Blasius boundary layer. The procedure to find the optimal wave packet was that of the linear global optimization theory, the optimization of the perturbation energy being performed for a laminar non-parallel boundary-layer flow without any assumption on the shape or on the frequency spectrum of the perturbation. The results showed that the optimal initial perturbation is characterized by a pair of streamwise-modulated counter-rotating vortices, tilted upstream, resulting at the optimal time in streak-like structures alternated in the streamwise direction. Such vortices trigger transition more effectively than streamwise-independent initial disturbances via a mechanism which goes through the formation of hairpin vortices.

Evidence for the presence of hairpin-shaped structures in transitional boundary layers (Wu \& Moin 2009; Cherubini et al. 2010b) proves that nonlinear mechanisms are indeed crucial in the transition scenario for wall-bounded shear flows. For parallel flows, such as the plane Couette flow and the flow in a circular pipe, the search for a purely nonlinear route to turbulence has been pursued in the last 20 years, after Waleffe (1995) demonstrated that the linear mechanism which yields streamwisehomogeneous streaks cannot easily trigger transition at low-to-moderate disturbance amplitude levels. Since the work of Nagata (1990), who found the first exact coherent solution of the Navier-Stokes (NS) equations for a Couette flow, followed by the theoretical work of Waleffe (1997, 1998), explaining the nature of the self-sustaining process responsible for maintaining such coherent structures, many authors advocated a theory in which transition and turbulence stem from the random walk of the systems trajectory in phase space among nonlinear mutually repelling states, which are exact unstable solutions of the NS equations (Kawahara \& Kida 2001; Faisst \& Eckhardt 2003; Wedin \& Kerswell 2004; Hof et al. 2004; Eckhardt et al. 2007; Schneider, Eckhardt \& Yorke 2007; Gibson, Halcrow \& Cvitanović 2009). Many such states have been identified, initially in small, periodic domains and very recently also as localized solutions in large domains (Duguet, Schlatter \& Henningson 2009; Mellibovsky et al. 2009; Schneider, Gibson \& Burke 2010a; Schneider, Marinc \& Eckhardt 2010b; Cherubini et al. 2011).

However, it is not yet clear which kind of initial perturbation is able to better switch on the process which brings the system most efficiently to these coherent states and then to turbulence. Recently, some studies have been carried out aimed at finding special initial disturbances, built by a linear combination of a limited number of 'basic modes', which cause the disturbed velocity field to approach such coherent structures (the lower-branch solution in a pipe flow in Viswanath \& Cvitanovic 2009, and the edge-state in a plane Couette flow in Duguet, Brandt \& Larsson 2010).

A more generic approach for identifying a purely nonlinear route to transition has been used by Pringle \& Kerswell (2010) for the pipe flow, and by Cherubini et al. (2010a) for the boundary-layer flow. For the case of the laminar boundary layer developing over a flat plate of interest here, the latter authors have used a global approach extending the linear transient growth analysis of Cherubini et al. $(2010 b)$ to the nonlinear framework. Optimizing the energy of the perturbations in a nonlinear framework, they have proved the existence of a nonlinear amplification mechanism of the disturbances which is more effective than the linear one and is capable of leading the flow to turbulence for lower values of the perturbation energy. 
The suitability of an energy optimization to determine the disturbances which bring the flow more effectively on the verge of turbulence has been confirmed by results obtained with a different optimization functional for the case of a Couette flow in a small domain (Monokrousos et al. 2011). For such a flow, using a functional constructed on thermodynamic considerations, better suited to target a turbulent state, has provided similar results to those obtained by using a disturbance energy functional. The optimizations performed by Pringle \& Kerswell (2010), Cherubini et al. (2010b) and Monokrousos et al. (2011) have provided a breakthrough on the importance of nonlinearity on the amplification mechanisms leading to turbulence. Nevertheless, for the case of the boundary-layer flow, it is still not clear whether the shape and the amplitude of the nonlinear optimal perturbations are robust features of the flow, as well as to what extent they depend on the Reynolds number, the domain length, the initial energy and/or the target time of the optimization. Moreover, still nothing is known about the mechanisms leading such optimal disturbances to turbulence, and the role of nonlinearity in the route to transition initiated by such fast growing perturbations is still to be identified. The present work aims at providing an answer to such questions by investigating: (i) the robustness of the nonlinear optimal perturbation and its dependence on the optimization parameters; (ii) the amplification mechanisms capable of triggering transition in a spatially developing boundary-layer flow following a purely nonlinear route.

A Lagrange multiplier technique is employed to find the optimal perturbation of given initial energy and Reynolds number $R e$ for the Blasius boundary-layer flow. The results of the optimization procedure are provided for two values of $R e$ and several values of the target time and the initial energy. For values of the initial energy larger than the threshold one, the optimal perturbation is found to be characterized by a fundamental invariant structure, the minimal seed for turbulent transition, which is formed by a localized array of vortices and low-momentum regions of typical length scale, capable of maximizing the energy growth the most rapidly. Furthermore, direct numerical simulations (DNSs) have been employed to study the mechanism of transition to turbulence when the flow is initialized using the minimal seed. Finally, a disturbance regeneration cycle is conceived, initiated by the fast growth and nonlinear evolution of the optimal disturbance, providing a possible scenario for the continuous regeneration of the same fundamental flow structures at smaller space and time scales.

This paper is organized as follows. In $\S 2$ we define the problem and describe the nonlinear optimization method. In $\S 3$, a thorough discussion of the results of the nonlinear optimization analysis is provided. In particular, in the first part, the focus is on the characterization of the optimal perturbation, whereas the second part deals with the optimal route to turbulence. Finally, the regeneration cycle is conjectured and concluding remarks are provided.

\section{Problem formulation}

\subsection{Governing equations and the numerical method}

The behaviour of a three-dimensional incompressible boundary-layer flow is governed by the NS equations:

$$
\begin{gathered}
\boldsymbol{u}_{t}+(\boldsymbol{u} \cdot \nabla) \boldsymbol{u}=-\nabla p+\frac{1}{\operatorname{Re}} \nabla^{2} \boldsymbol{u}, \\
\nabla \cdot \boldsymbol{u}=0
\end{gathered}
$$


where $\boldsymbol{u}$ is the velocity vector and $p$ is the pressure term (including the contribution of conservative-force fields). Dimensionless variables are defined with respect to the inflow boundary-layer displacement thickness, $\delta^{*}$, and the free stream velocity, $U_{\infty}$, so that the Reynolds number is $R e=U_{\infty} \delta^{*} / v, v$ being the kinematic viscosity. Several computational domains have been employed, the reference domain having dimensions equal to $L_{x}=200, L_{y}=20$ and $L_{z}=10.5, x, y$ and $z$ being the streamwise, wall-normal and spanwise directions, respectively. The Blasius base flow is obtained by integrating the NS equations with the following boundary conditions: at inlet points, placed at $x_{i n}=200$ downstream of the leading edge of the flat wall, a Blasius boundary-layer profile is imposed for the streamwise and wall-normal components of the velocity vector whereas the spanwise component is set to zero. At outlet points, placed at $x_{\text {out }}=400$ for the reference domain, a standard convective condition is employed (Bottaro 1990). At the bottom wall the no-slip boundary condition is prescribed. At the upper-boundary points the Blasius solution is imposed for the wall-normal component of the velocity, whereas the spanwise velocity component and the spanwise vorticity are set to zero. Finally, in the spanwise direction periodicity is imposed for the three velocity components. The NS equations are discretized by a finite-difference fractional-step method using a staggered grid (Verzicco \& Orlandi 1996). A secondorder accurate centred space discretization is used. After a grid-convergence analysis, a mesh made up by $901 \times 150 \times 61$ points, clustered towards the wall so that the thickness of the first cell close to the wall is equal to 0.1 , is selected for the reference domain.

\subsection{Nonlinear optimization}

The nonlinear behaviour of a perturbation $\boldsymbol{q}=\left(u^{\prime}, v^{\prime}, w^{\prime}, p^{\prime}\right)^{\mathrm{T}}$ evolving in a laminar incompressible flow over a flat plate is studied by employing the NS equations written in a perturbative formulation, with respect to the two-dimensional Blasius steady-state solution, $\boldsymbol{Q}=(U, V, 0, P)^{\mathrm{T}}$. A zero-perturbation condition is chosen for the three velocity components at the $x$-constant and $y$-constant boundaries, whereas periodicity of the perturbation is imposed in the spanwise direction. The zero-perturbation condition at inflow and outflow points is enforced by means of a fringe region (Cherubini et al. 2010b), which allows the perturbation at the exit boundary to vanish smoothly.

In order to find the perturbation at $t=0$ providing the largest disturbance growth at a given target time, T, a Lagrange multiplier technique is used (Zuccher, Luchini \& Bottaro 2004; Pringle \& Kerswell 2010). The disturbance energy density is defined as

$$
\begin{aligned}
E(t) & =\int_{0}^{L_{z}} \int_{o}^{L_{y}} \int_{x_{\text {in }}}^{x_{\text {out }}}\left[u^{\prime 2}(t)+v^{\prime 2}(t)+w^{\prime 2}(t)\right] \mathrm{d} x \mathrm{~d} y \mathrm{~d} z \\
& =\int_{V}\left[u^{\prime 2}(t)+v^{\prime 2}(t)+w^{\prime 2}(t)\right] \mathrm{d} V ;
\end{aligned}
$$

the aim is to find the initial perturbation $\boldsymbol{q}_{0}$ of given initial energy $E(0)=E_{0}$ which can induce at target time $T$ the largest energy $E(T)$. Thus, the objective function of the procedure, $G$, is the energy gain $G=E(T) / E(0)$. The Lagrange multiplier technique consists of searching for extrema of an augmented functional, $\mathscr{L}$, with respect to every independent variable, the three-dimensional incompressible NS equations and the value of the initial energy being imposed as constraints. The gradient of the augmented functional with respect to every independent variable is forced to vanish by means of a coupled iterative approach similar to that used by Zuccher et al. (2004) and Pringle \& 


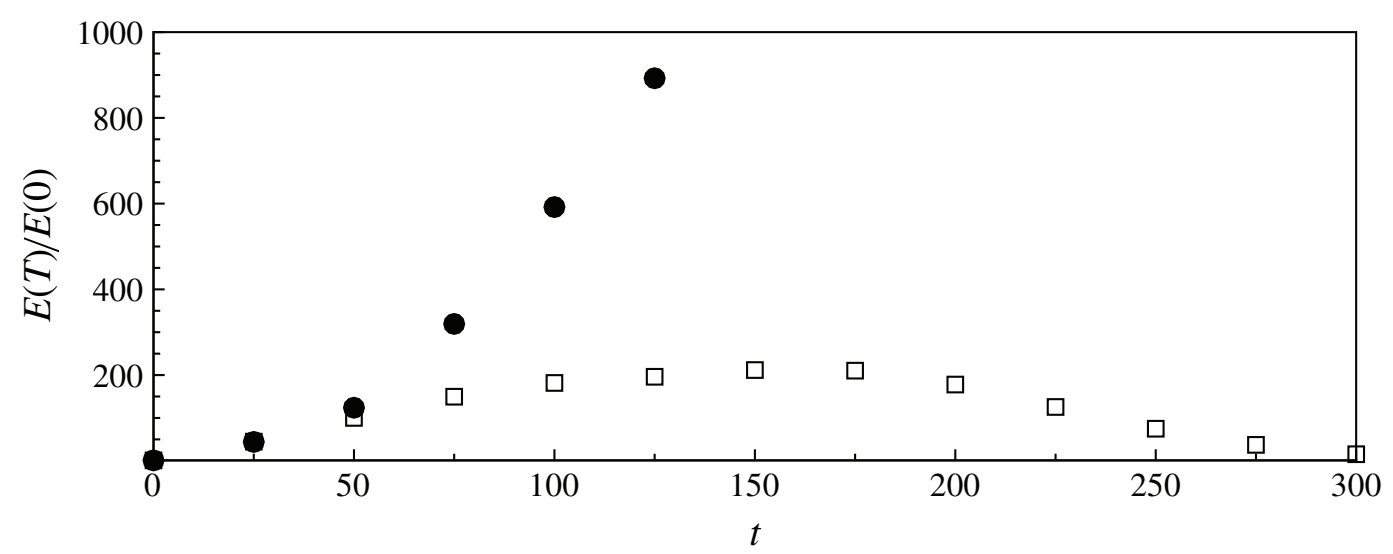

FIGURE 1. Optimal energy gain versus target time $T$ for $R e=300, E_{0}=0.1$. The white squares indicate the results of a linear optimization; the black circles indicate the nonlinear results.
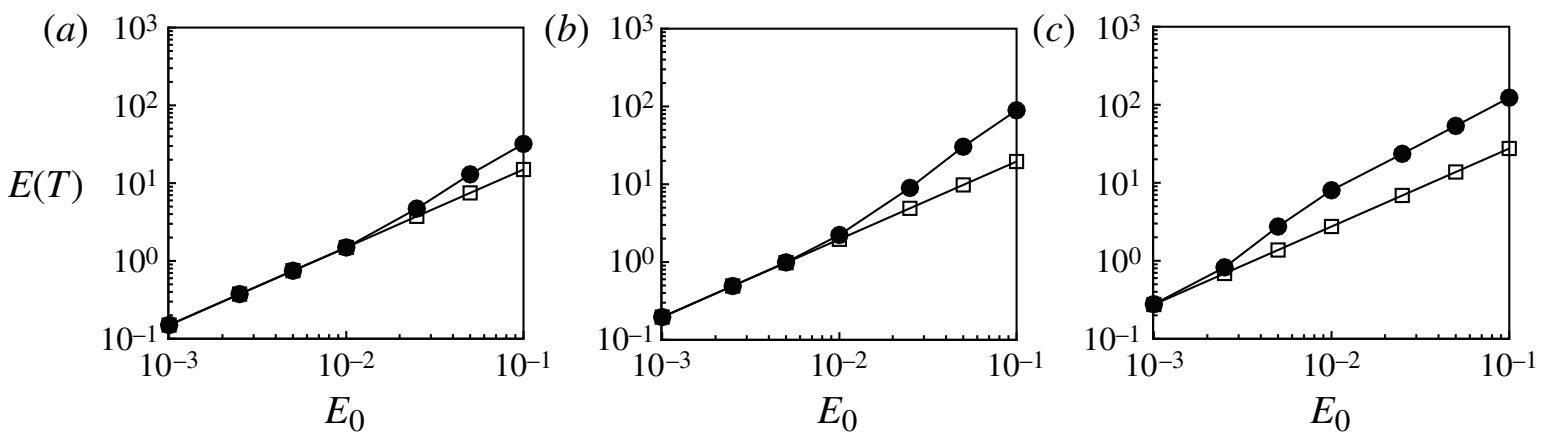

FIgURE 2. Optimal energy at target time $T$ for $(a) R e=300, T=75 ;(b) R e=300, T=125$; (c) $R e=610, T=75$. The white squares indicate the results of a linear optimization; the black circles indicate the nonlinear results.

Kerswell (2010), employing a conjugate gradient method. A detailed description of the optimization technique is provided in Appendix.

\section{Results}

\subsection{Nonlinear optimal perturbations}

The nonlinear optimization has been performed for two values of the Reynolds number; the first, $R e=300$, is subcritical with respect to Tollmien-Schlichting waves, whereas the second, $R e=610$, is supercritical. Figure 1 shows the value of the optimal energy gain versus the target time for $R e=300$ and $E_{0}=0.1$ (black circles). For comparison, the optimal energy gain obtained by the corresponding linear optimization (see Cherubini et al. 2010b) is provided in the same figure (white squares). For $T>50$, the nonlinear optimal energy gain is remarkably larger than the corresponding linear one. In particular, the energy gain grows in time following a quasi-exponential curve, unlike the linear case which shows an initial growth phase followed by a decay. The trend of the energy gain curve obtained for $R e=300$ is similar to that obtained for $R e=610$ (see figure 2 in Cherubini et al. 2010a). However, a higher increase of the gain is obtained for $R e=610$ with respect to $R e=300$. The influence of the parameter $E_{0}$ on the value of the optimal energy is shown in figure 2, for two values of the target time and of the Reynolds number. In all cases, a threshold on the initial energy exists (hereafter called the nonlinearity threshold) 
$t=0, R e=610$
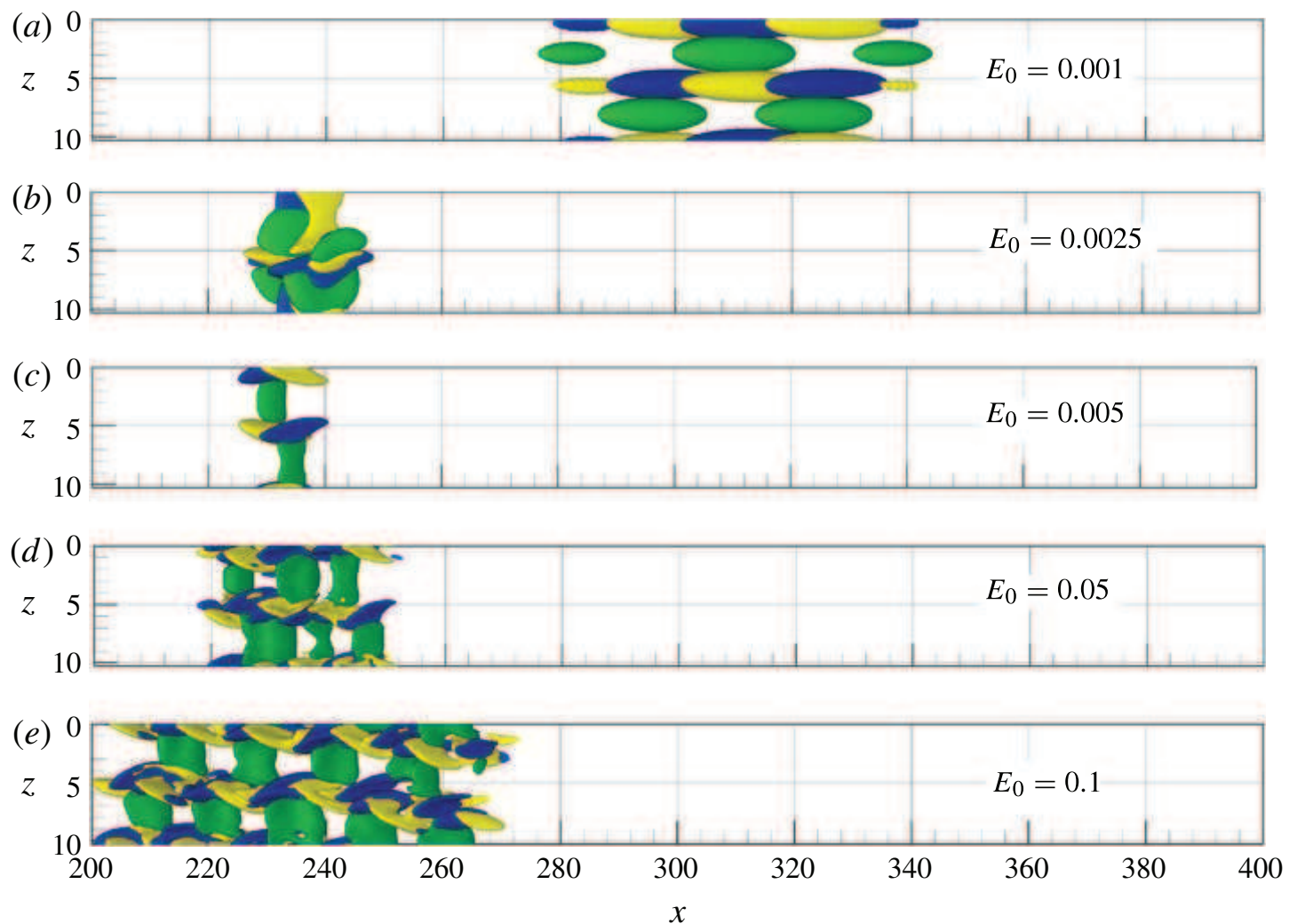

FIGURE 3. (Colour online available at journals.cambridge.org/flm) Initial perturbations obtained by the nonlinear optimization for $R e=610$ and target time $T=75$ : isosurfaces of the optimal perturbations (grey (green online) for the negative streamwise component; dark and light (blue and yellow online) for negative and positive streamwise vorticity, respectively) with initial energy (from $a$ to $e$ ) $E_{0}=0.001$ (surfaces for $u^{\prime}=-0.0005, \omega_{x}^{\prime}= \pm 0.005$ ), $E_{0}=0.0025\left(u^{\prime}=-0.001, \omega_{x}^{\prime}= \pm 0.01\right), E_{0}=0.005\left(u^{\prime}=-0.007, \omega_{x}^{\prime}= \pm 0.03\right), E_{0}=0.05$, and $E_{0}=0.1\left(u^{\prime}=-0.01, \omega_{x}^{\prime}= \pm 0.05\right)$. Axes are not on the same scale.

from which strong modifications are observed in the nonlinear optimal energy with respect to the linear one. Such a threshold decreases when the Reynolds number or the target time increase (as one can observe by inspection of figure 2). Moreover, crossing such a threshold yields strong modifications in the shape of the optimal perturbations. This is clearly shown in figure 3, which provides the optimal initial perturbations obtained for $R e=610$ and $T=75$, for five values of the initial energy, $E_{0}$. For the lowest value, $E_{0}=0.001$ (top frame), the perturbation is similar to that obtained by the linear optimization (see Cherubini et al. 2010b); it is characterized by elongated vortices aligned along $x$, localized in the middle of the domain. For $0.001<E_{0}<0.005$, the shape of the optimal perturbation changes remarkably, moving close to the inlet, and decreasing its streamwise size. For $E_{0} \geqslant 0.005$, the structure of the optimal perturbation changes slightly, being characterized by a basic building block (cf. Cherubini et al. 2010a), which is replicated one or more times along $x$ and/or $z$ for increasing values of the initial energy. The same basic building block is observed for larger target times, for values of the initial energy larger than the nonlinearity threshold, and will henceforth be called the minimal seed, i.e. the smallest structure by which the maximum energy growth is achieved over short times. It is characterized by alternated vortices inclined with respect to the streamwise direction (light and dark surfaces, yellow and blue online, indicating the positive and negative 

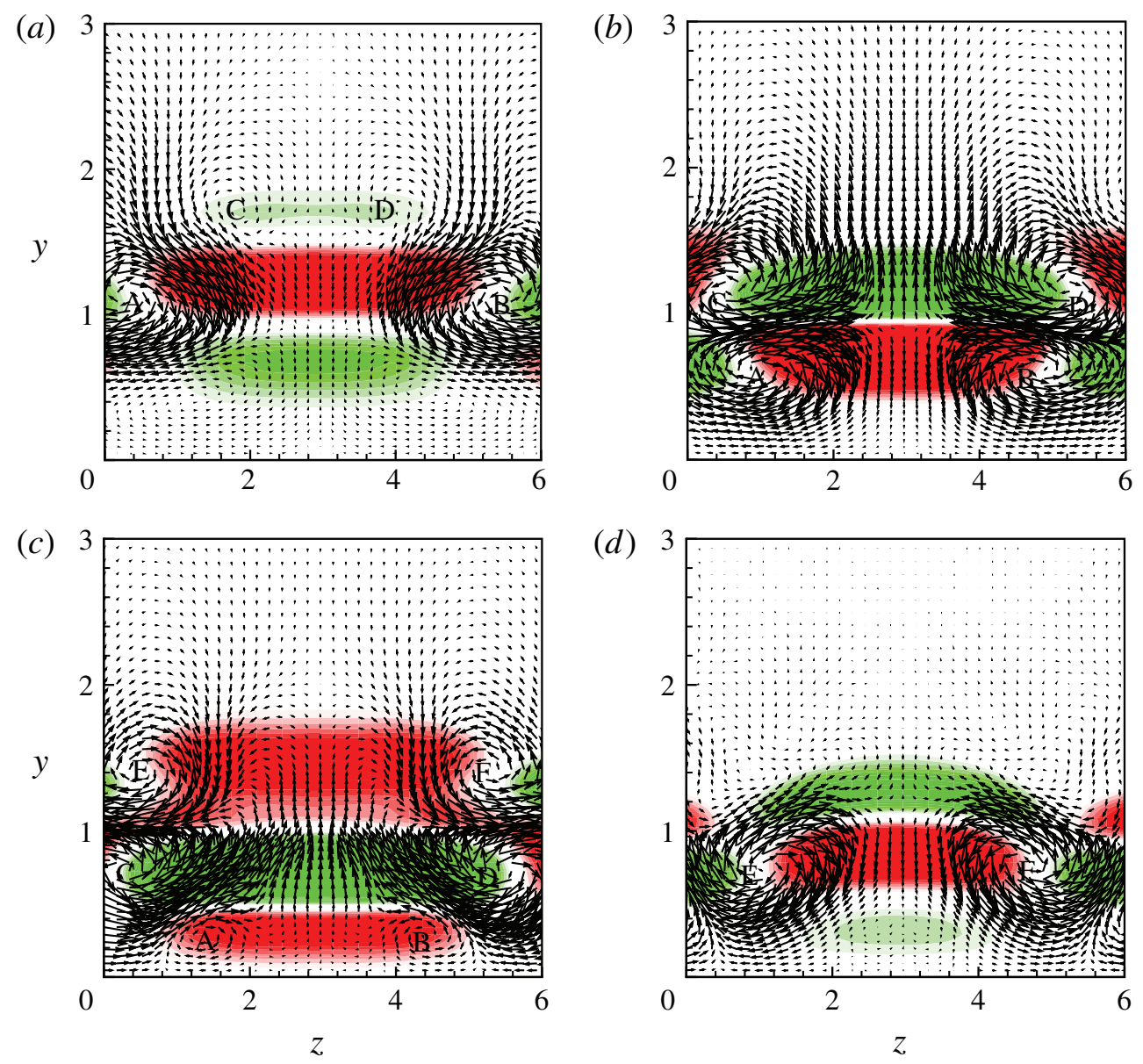

FIGURE 4. (Colour online) Contours and vectors of the velocity components of the initial optimal perturbation obtained for $R e=610, T=75$, and $E_{0}=0.01$, on the planes $x=224(a)$, $x=228(b), x=232(c)$ and $x=236(d)$. Shaded contours indicate the streamwise disturbance velocity (dark (red online) for positive values; light (green online) for negative values), vectors represent the wall-normal and the spanwise disturbance velocity components. Axes are not on the same scale.

streamwise vorticity, $\omega_{x}^{\prime}$, respectively), which lay on the flanks of a region of negative streamwise velocity disturbance (grey surfaces, green online). The inclined vortices are shown in figure 4 , which provides four $x$-constant sections of the optimal initial perturbation obtained for $T=75, E_{0}=0.01$ and $R e=610$. The vortices are inclined with respect to the mean flow, both in the wall-normal and in the spanwise direction. The upstream tilting with respect to the wall-normal direction, which can be observed in figure $4(a, b)$ for the vortices $\mathrm{A}$ and $\mathrm{B}$, in figure $4(b, c)$ for the vortices $\mathrm{C}$ and $\mathrm{D}$, and in figure $4(c, d)$ for the vortices $\mathrm{E}$ and $\mathrm{F}$, is linked to the Orr mechanism (see Schmid \& Henningson 2001). This inclination is observed also in the linear optimal case, as shown by Cherubini et al. (2010b) and Monokrousos et al. (2010), due to the fact that a transient energy growth is produced when the mean flow tilts downstream the structures initially opposing the base flow. On the other hand, spanwise tilting is not observed in the linear case, in which the optimal perturbations are characterized by elongated vortices perfectly aligned with the streamwise direction (see figure 3a). Another remarkable difference with respect to the linear optimal perturbation concerns the relative magnitude of the velocity components. Figure 5 provides a section of the flow at $y=1.4$ showing the contours of the streamwise (shaded), wall-normal (black lines) and spanwise (white lines) velocity components 


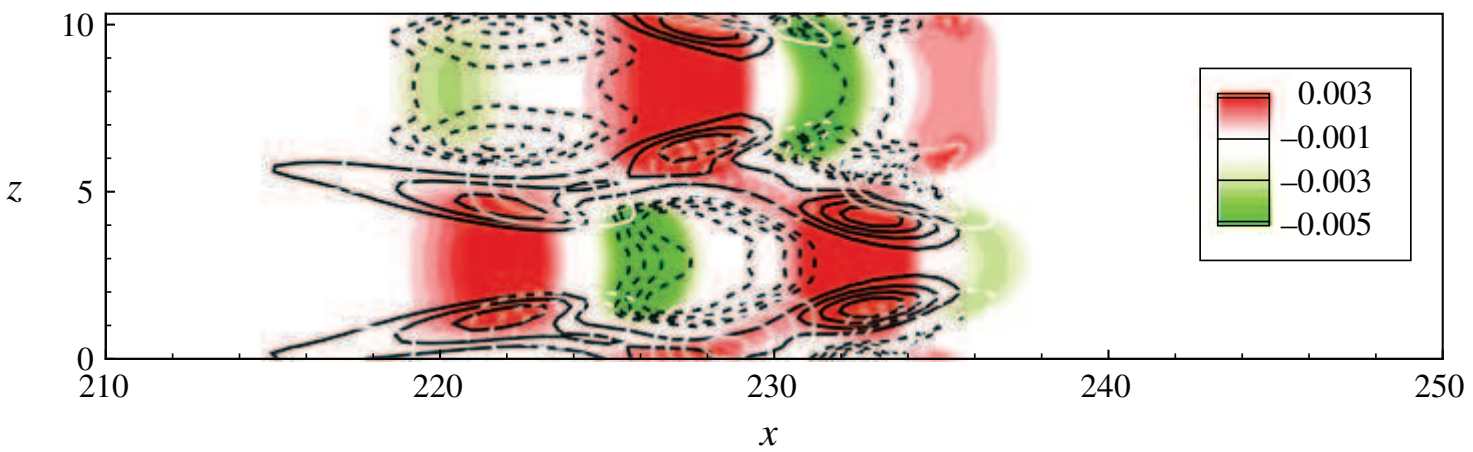

FIGURE 5. (Colour online) Section at $y=1.4$; contours of the velocity components of the initial optimal perturbation obtained for $R e=610, T=75$, and $E_{0}=0.01$. Shaded contours indicate the streamwise disturbance velocity component (light, green online, for negative values; dark, red online, for positive ones), black and white lines represent the wall-normal and the spanwise disturbance velocity components, respectively (solid for negative values, dashed for positive values). The values of the contours are $v^{\prime}=-0.004,-0.003,-0.002,-0.001,0.001,0.0015,0.002,0.0025$ and $w^{\prime}=$ $\pm 0.01,0.008,0.006,0.004,0.002$. Axes are not on the same scale.

of the initial optimal perturbation obtained for $T=75, R e=610$ and $E_{0}=0.01$. The component of the initial velocity perturbation having the highest absolute value is the streamwise component $\left(u_{\max }^{\prime-}=-0.018, u_{\max }^{\prime+}=0.011\right)$, unlike the linear case in which the streamwise component is the smallest ( $u_{\max }^{\prime}= \pm 0.00026$, not shown). Moreover, unlike the linear optimal case, at the initial time, regions with high negative streamwise component of the velocity disturbance are associated with high positive values of the wall-normal component (compare the dashed lines with the grey (green online) regions in figure 5, as well as the upwards arrows with the grey (green online) regions in figure 4).

What comes out from the optimal perturbations at $T$ is shown in figure 6 , for the same five values of the initial energy used in figure 3. One can observe $\Lambda$-shaped low-momentum structures, along with streamwise inclined vortices tilted downstream. Such structures are observed for all of the initial energies larger than the nonlinearity threshold, although one can notice that, when the initial perturbation occupies more space in the spanwise and streamwise direction, the minimal seeds interact nonlinearly, leading to more chaotic, small-scale structures over a finite time (see, in particular, the bottom frame of figure 6).

Similar optimal perturbations are obtained at different target times and Reynolds numbers, when the initial energy is larger than the corresponding nonlinearity threshold. As an example, figure 7 shows the streamwise component of the velocity perturbation (grey (green online) surfaces) as well as the streamwise vorticity perturbation (light and dark (yellow and blue online) surfaces) for the optimal perturbation obtained for $R e=300, T=125$ and $E_{0}=0.01$. One can observe two minimal seeds (top frame), having the same structure and a similar spatial extent as those found at $R e=610$ (see figure 3), with inclined vortices staggered in $x$. They are tilted downstream by means of the Orr mechanism, yielding at larger time $\Lambda$ structures and vortices with a finite (positive) inclination with respect to $x$ (second frame).

It is important to establish whether the optimal perturbation maintains its characteristic shape and size varying the streamwise or spanwise domain length. Thus, further optimizations have been performed for different streamwise and spanwise domain lengths, maintaining the same local grid resolution of the previous case 


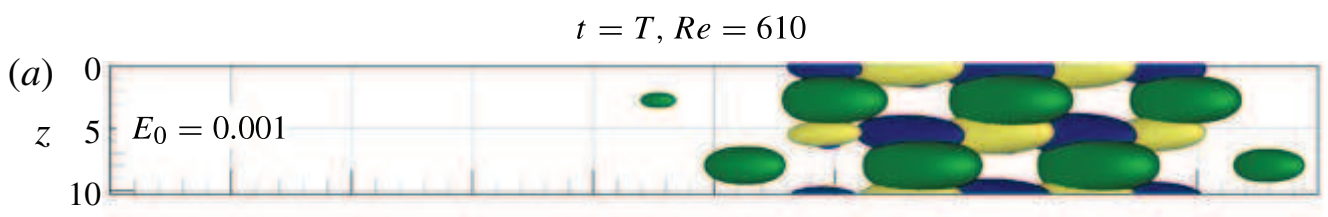

(b)
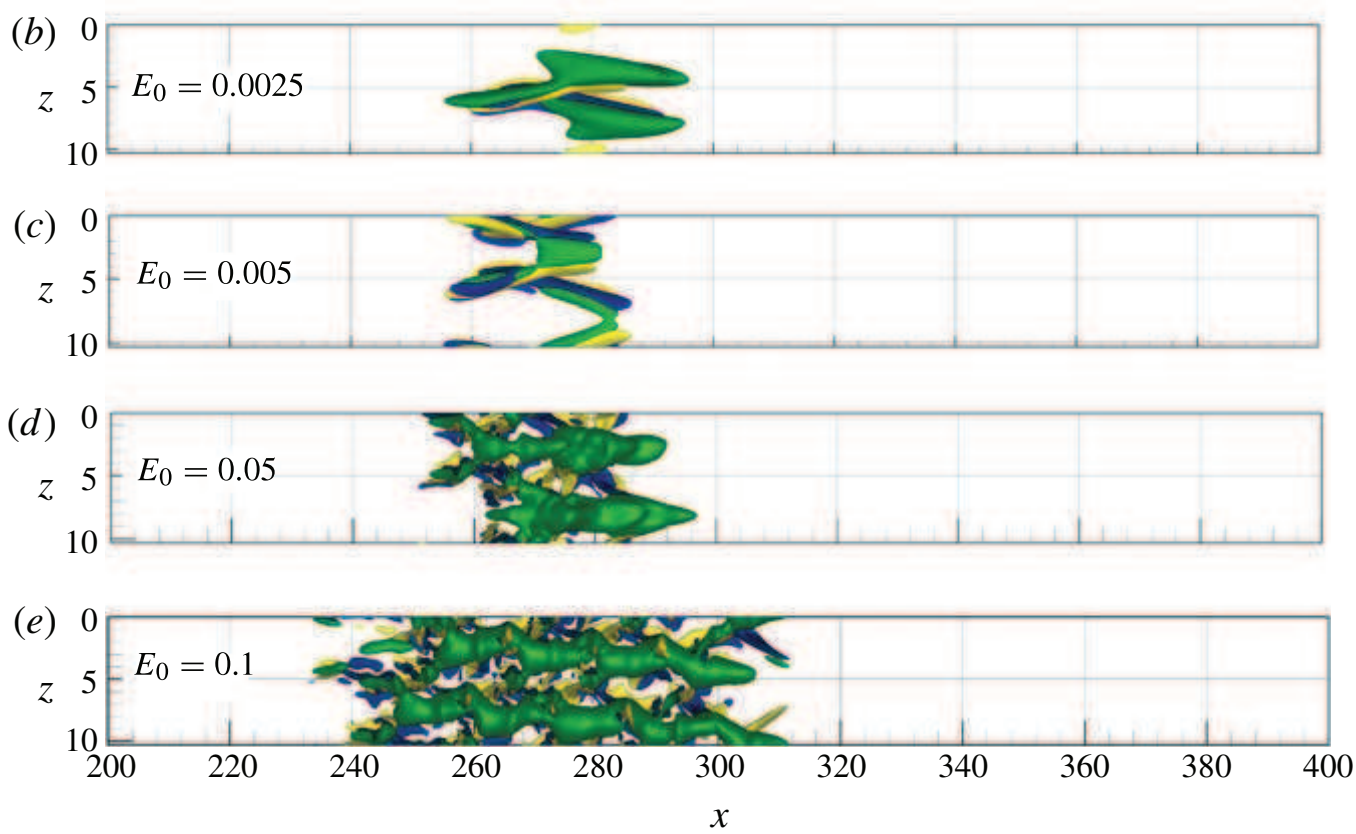

Figure 6. (Colour online) Outcome at $t=T=75$ and $R e=610$ of the optimal initial perturbations injected at $t=0$ : isosurfaces of the perturbations (grey (green online) for the negative streamwise velocity component; dark and light (blue and yellow online) for negative and positive streamwise vorticity, respectively) with initial energy (from $a$ to $e$ ) $E_{0}=0.001$ (surfaces for $\left.u^{\prime}=-0.01, \omega_{x}^{\prime}= \pm 0.015\right), E_{0}=0.0025\left(u^{\prime}=-0.02, \omega_{x}^{\prime}= \pm 0.05\right), E_{0}=0.005$ $\left(u^{\prime}=-0.08, \omega_{x}^{\prime}= \pm 0.08\right), E_{0}=0.05$ and $E_{0}=0.1\left(u^{\prime}=-0.2, \omega_{x}^{\prime}= \pm 0.5\right)$. Axes are not on the same scale.
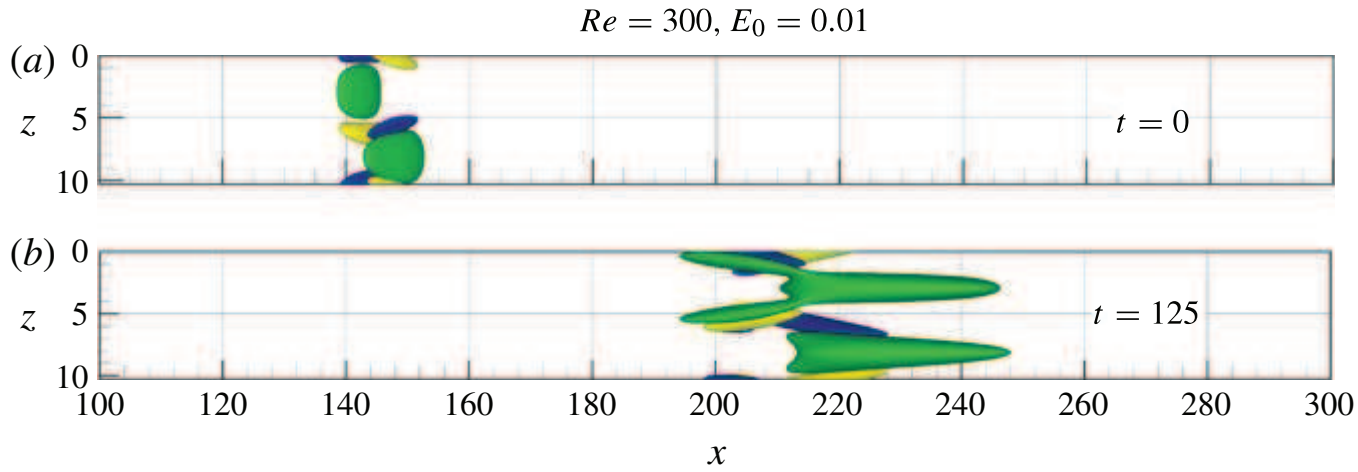

FIgURE 7. (Colour online) Time evolution of the optimal perturbation obtained by the nonlinear optimization for $T=125, E_{0}=0.01$ and $R e=300$, at time $t=0(a)$ and $t=T(b)$. The grey (green online) surfaces indicate the negative streamwise component of velocity $\left(u^{\prime}=-0.006\right.$ at $t=0, u^{\prime}=-0.04$ at $\left.t=T\right)$, the dark and light (blue and yellow online) surfaces indicate negative and positive streamwise vorticity, respectively $\left(\omega_{x}^{\prime}= \pm 0.04\right)$. Axes are not on the same scale.

(namely, the number of points in the streamwise or in the spanwise direction is scaled with $L_{x}$ or $L_{z}$, respectively). Optimizations performed with streamwise domain lengths $L_{x}=100$ and $L_{x}=400$ (for $R e=610, E_{0}=0.01$ and $T=75$ ), indicate that 
(a)

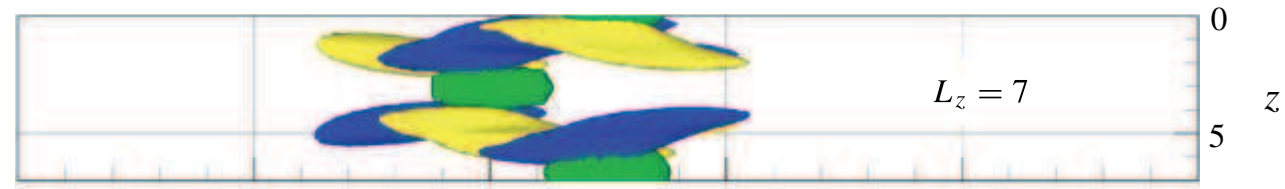

(b)

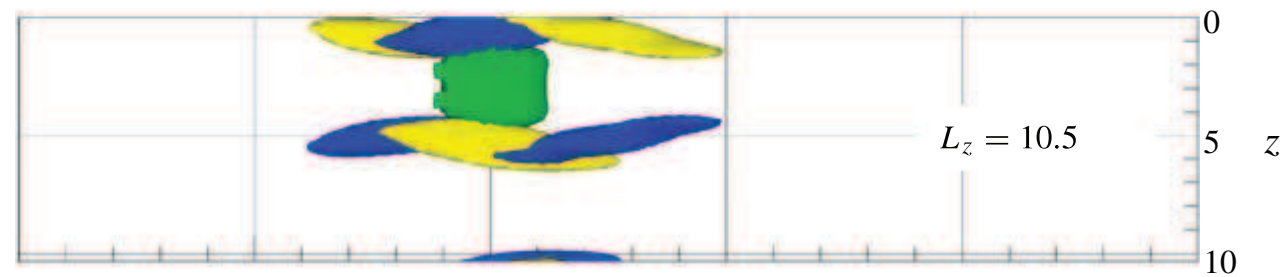

(c)

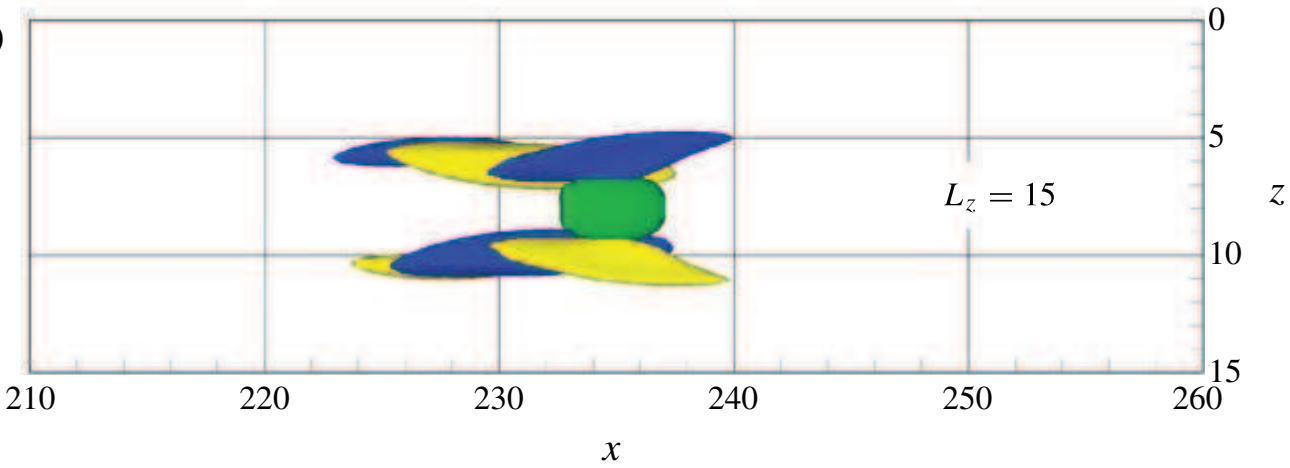

FIGURE 8. (Colour online) Initial optimal perturbations obtained by the nonlinear optimization at $T=75, E_{0}=0.01$ and $R e=610$, for three different spanwise domain lengths, $L_{z}=7(a), L_{z}=10.5(b)$ and $L_{z}=15(c)$. The grey (green online) surfaces indicate the negative streamwise component of velocity $\left(u^{\prime}=-0.01\right)$; the dark and light (blue and yellow online) surfaces indicate negative and positive streamwise vorticity, respectively $\left(\omega_{x}^{\prime}= \pm 0.05\right)$.

the optimal disturbance is practically independent of the streamwise domain length. Similarly, optimizations carried out with $L_{z}=15$ and $L_{z}=7$ show that the optimal perturbation and energy gain is slightly dependent on the spanwise domain size, the variation on the optimal energy gain being lower than $10 \%$. However, it is noteworthy that the shape of the minimal seed is qualitatively unchanged. Figure 8 shows the initial optimal disturbances obtained at $R e=610, E_{0}=0.01$ and $T=75$ for the three values of the spanwise domain length considered here: the shape of the perturbation remains the same. Moreover, the spanwise extent of the optimal perturbation varies only slightly with $L_{z}$, hinting at the fact that the spanwise length scale selected by the optimizations is a robust feature of the problem.

The persistence of the minimal seed structure at different values of the initial energy, Reynolds number, domain sizes and target times indicates that the structure obtained here, which maximizes the disturbance energy over a finite time, has an intrinsic fundamental importance for the boundary layer.

\subsection{The route of the minimal seed to turbulence}

\subsubsection{Description of the overall transition process}

In this section, we study in detail the route to turbulence of the minimal-seed perturbation, employing the DNS. For most of the computations the minimal seed obtained for $T=75, E_{0}=0.01$ and $R e=610$ has been used to initialize the flow field. Computations have been performed in a domain three times longer in $x$ than that used for the optimizations, in order to follow the flow evolution for a sufficiently long 


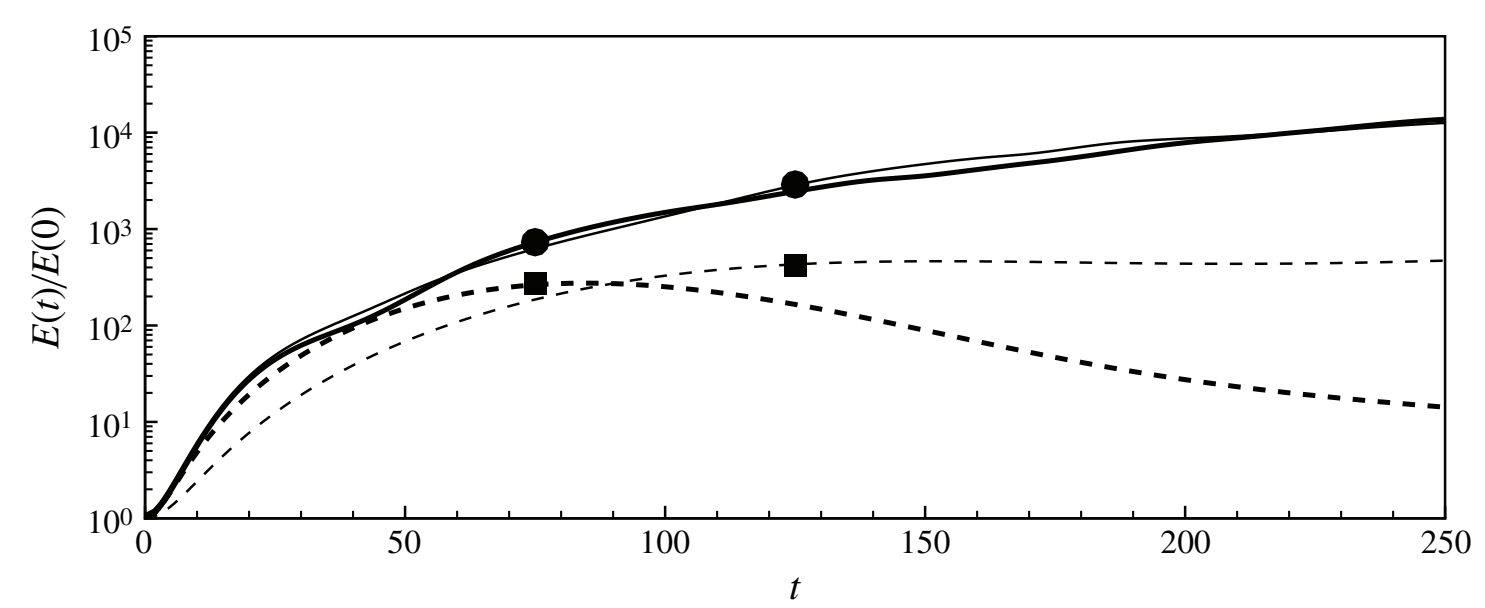

FIGURE 9. Energy gain versus time obtained by DNSs initialized using the linear (dashed lines) and nonlinear (solid lines) optimal perturbations computed for $T=75$ (thick lines) and $T=125$ (thin lines), $E_{0}=0.01$ and $R e=610$. The black squares and circles represent the energy gain obtained by the linear and nonlinear optimization, respectively.

time before the disturbance wave packet leaves the domain. A $2701 \times 150 \times 61$ grid has been used, so that the local grid resolution is the same of the optimization runs. Figure 9 provides the energy gain computed by DNSs initialized with four optimal perturbations, two linear and two nonlinear, obtained for two target times, and for the same value of the initial energy, $E_{0}=0.01$. Both nonlinear optimal perturbations grow very rapidly in time, reaching an energy gain close to 8000 in $\sim 200$ time units. On the other hand, the linear optimal perturbations do not show a rapid energy growth: that obtained for the smaller value of $T$ starts to decay right after the target time (for $t \approx 85$ ); the other experiences saturation for a considerable amount of time followed by decay (at $t \approx 475$, not shown). Evaluating the spanwise-averaged skin friction coefficient $C_{f}$, we have verified that when the DNS is initialized by the minimal seed perturbation with $E_{0}=0.01, R e=610$ and $T=75$, the $C_{f}$ reaches values which are typical of turbulent flows for $t>200$ (see figure 5 in Cherubini et al. 2010a); on the other hand, when the simulation is initialized by the optimal perturbation resulting from a linear optimization with the same initial energy and target time, $C_{f}$ reaches values which are at most $1.5 \%$ larger than those of the laminar reference curve (not shown). Thus, for the same value of the initial energy, the nonlinear optimal perturbation is able to lead the flow to turbulence, whereas the linear perturbation is not. Optimal perturbations obtained for larger initial energies are even more efficient in inducing transition. Indeed, observing in figure 10 the skin friction coefficient corresponding to the evolution of the nonlinear optimal perturbation obtained for $T=75$ with $E_{0}=0.1$, one can conclude that transition to turbulence occurs earlier in space and time with respect to the case with $E_{0}=0.01$ (cf. the results in Cherubini et al. 2010a). In particular, turbulence is reached at the target time, whereas for the lower value of $E_{0}$ transition is reached $\sim 175$ time units after the target time. In both cases one can notice the presence of an elongated calmed region localized at the trailing edge of the wave packet, a common feature of turbulent spots.

It is thus interesting to investigate the route to turbulence followed by the minimal seed. A qualitative picture of the transition process initiated by the nonlinear optimal perturbation has been given by Cherubini et al. (2010a). In the present paper we provide a more detailed analysis of the transition route which was first sketched 


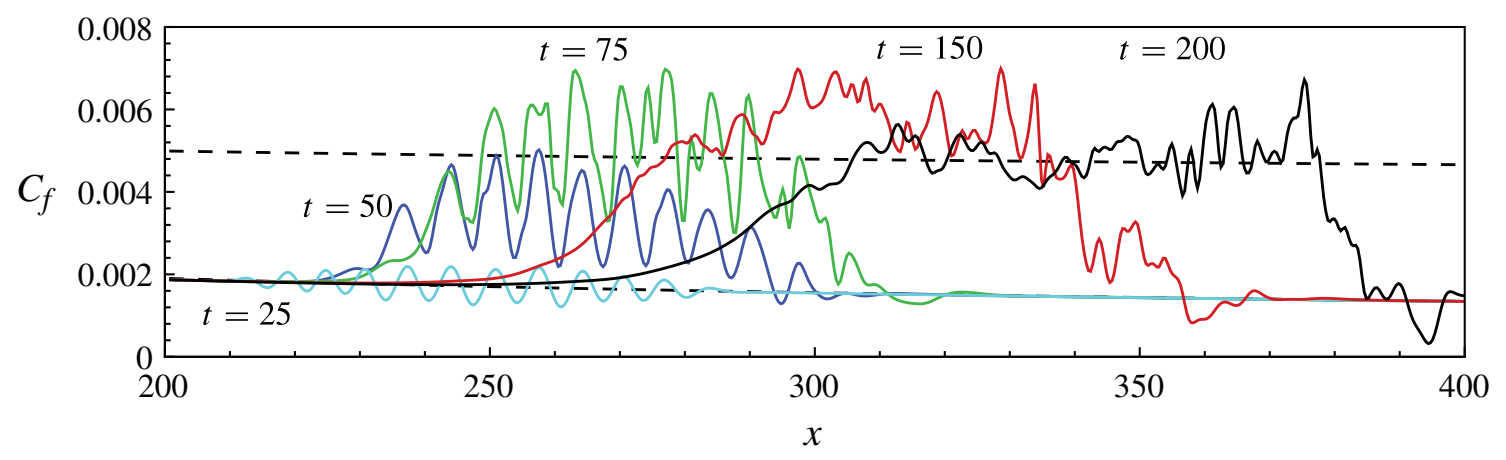

FIGURE 10. (Colour online) Streamwise distribution of the spanwise-averaged skin-friction coefficient extracted at $t=25,50,75,150,200$ (solid lines from left to right) from a DNS initialized by the nonlinear optimal perturbation obtained for $T=75, E_{0}=0.1$ and $R e=610$. The laminar and turbulent distributions of $C_{f}$ (bottom and top dashed lines, respectively) are also reported for comparison.

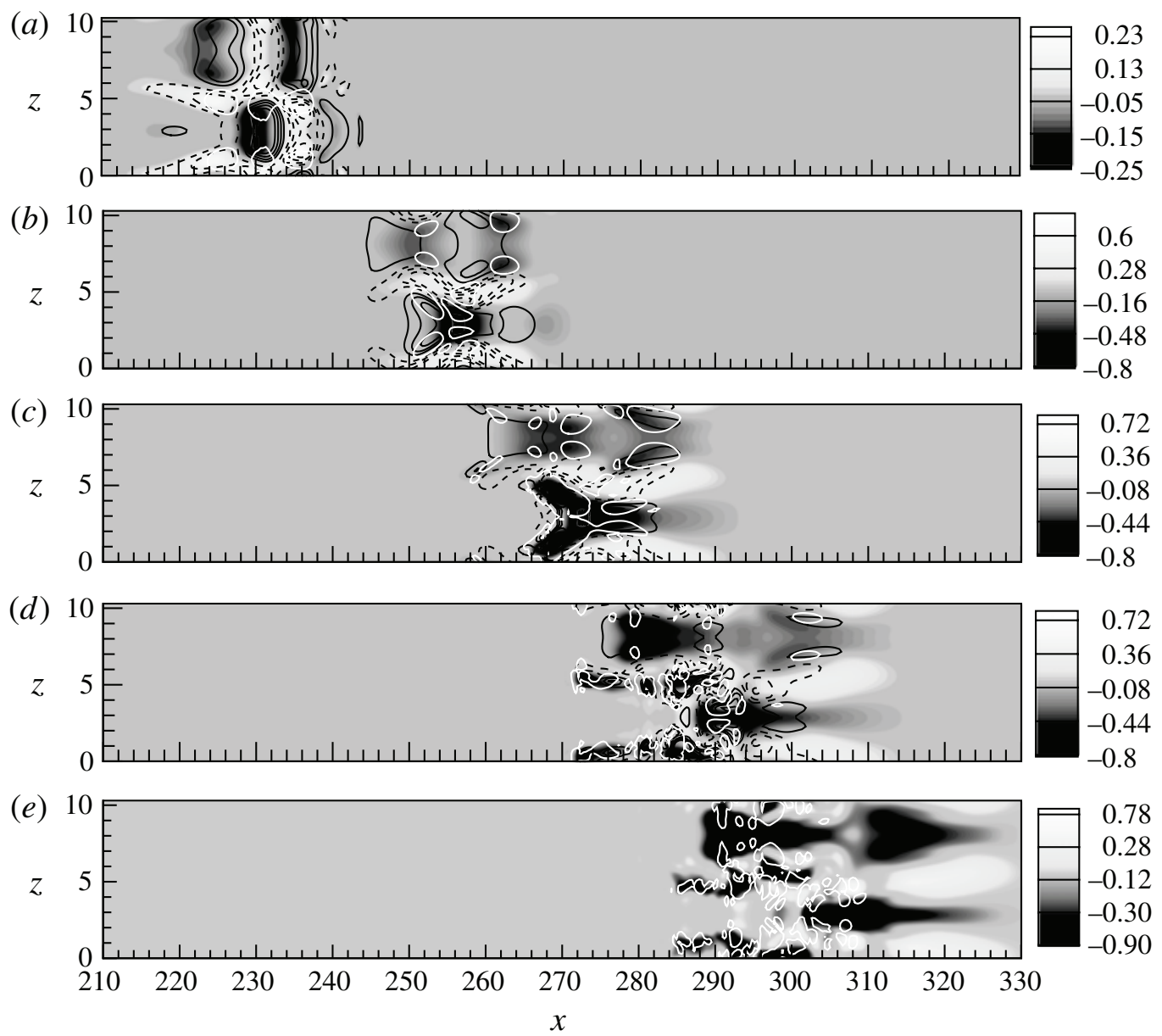

FIGURE 11. Snapshots of the perturbation on the plane $y=1.8$ at $t=0,35,65,95$ and 125 (from $a$ to $e$ ) obtained by the DNS initialized by the nonlinear optimal for $T=75$, $R e=610$ and $E_{0}=0.01$. The shaded contours refer to the streamwise component of the velocity perturbation, the solid and dashed black lines to the negative and positive values of the wall-normal velocity component, respectively, and the white lines to the $Q$ criterion. Each variable has been normalized using its maximum value at each time; solid and dashed contours show the values $Q=0.02$ and $v^{\prime}= \pm[0.1,0.2,0.3,0.4]$. 
in our previous paper. Figure 11 shows the contours of the streamwise and wallnormal components of the velocity perturbation, as well as of the $Q$ criterion (Hunt, Wray \& Moin 1988), on the $y=1.8$ plane at $t=0,35,65,95,125$ (to allow a comparison among several times, each variable has been normalized using its maximum value at each time). One can observe that the $\Lambda$ vortices at $t=35$ (white contours on the second frame) almost overlap on the $\Lambda$-shaped low-momentum zones. This is a completely different picture from the linear case. It is known that in a linear framework the amplification of the optimal perturbation is mainly due to the transport of the base flow momentum by a pair of streamwise vortices (the lift-up effect), which induces streamwise streaks at a finite time. Thus, the bypass route to transition goes through secondary instability of the streaks and nonlinear mixing which sustains the streamwise vortices. On the other hand, in the present case, the optimal perturbation is characterized by streamwise-inclined vortices; they transport the flow momentum causing an amplification of the streamwise component of velocity along them and inducing the creation of low- and high-momentum zones modulated in $x$, i.e. $\Lambda$-shaped low-momentum zones and sinuous high-momentum zones. Owing to such a streamwise modulation of the momentum, the flow can bypass the mechanism of secondary instability and reach transition via a more rapid route.

Moreover, it is worth pointing out that both the base flow momentum and the finite amplitude initial streamwise perturbation are transported by the inclined vortices, inducing defects in the base flow and dislocations of the initial disturbance. Here, defects are defined as modifications of the base flow with zero temporal frequency, which can affect the stability properties of the base flow (cf. Biau \& Bottaro 2009), whereas dislocations are defined as regions of strong interaction between neighbouring flow structures of finite amplitude, resulting in the merging or splitting of the initial structures. Such flow modifications are mostly due to the spatial correlation of regions of high streamwise velocity with regions of low wall-normal velocity components, and vice versa, as can be observed in figure 11. Therefore, the low-momentum perturbation is rapidly transported up in the boundary layer, whereas the highmomentum perturbation is convected close to the wall. Such effects can be clearly observed looking at planes perpendicular to the streamwise direction (figure 12), providing the contours and vectors of the disturbance velocity components of the optimal perturbation at $t=75$. Comparing it with figure $4(t=0)$, one can notice that the regions of negative streamwise perturbation are lifted up in the wall-normal direction (light (green online) contours), whereas the positive streamwise perturbations (dark (red online) contours) plunge towards the wall. As a result, the horizontal shear layers present in figure 4 increase in magnitude and change in shape, inducing strong modifications and inflection points in the base flow profile. The shapes of the lowand high-momentum zones are strongly reminiscent of those characterizing Görtler and Dean vortices (Guo \& Finlay 1991; Bottaro 1993) while undergoing an Eckhaus instability.

The creation of inflection points can be more clearly observed in figure 13, showing the profiles of the instantaneous streamwise velocity at three times and positions within the flow, along with the isosurfaces of the streamwise perturbation velocity at each time. An inflection point is firstly established in the flow at $t=75$, when dislocations have already formed; then, at larger time, $t=100$, the main $\Lambda$ structure breaks up into smaller disturbance patches. This suggests that the inflection points of the mean-flow profile are related to the rupture of large-scale structures into smallerscale structures. 

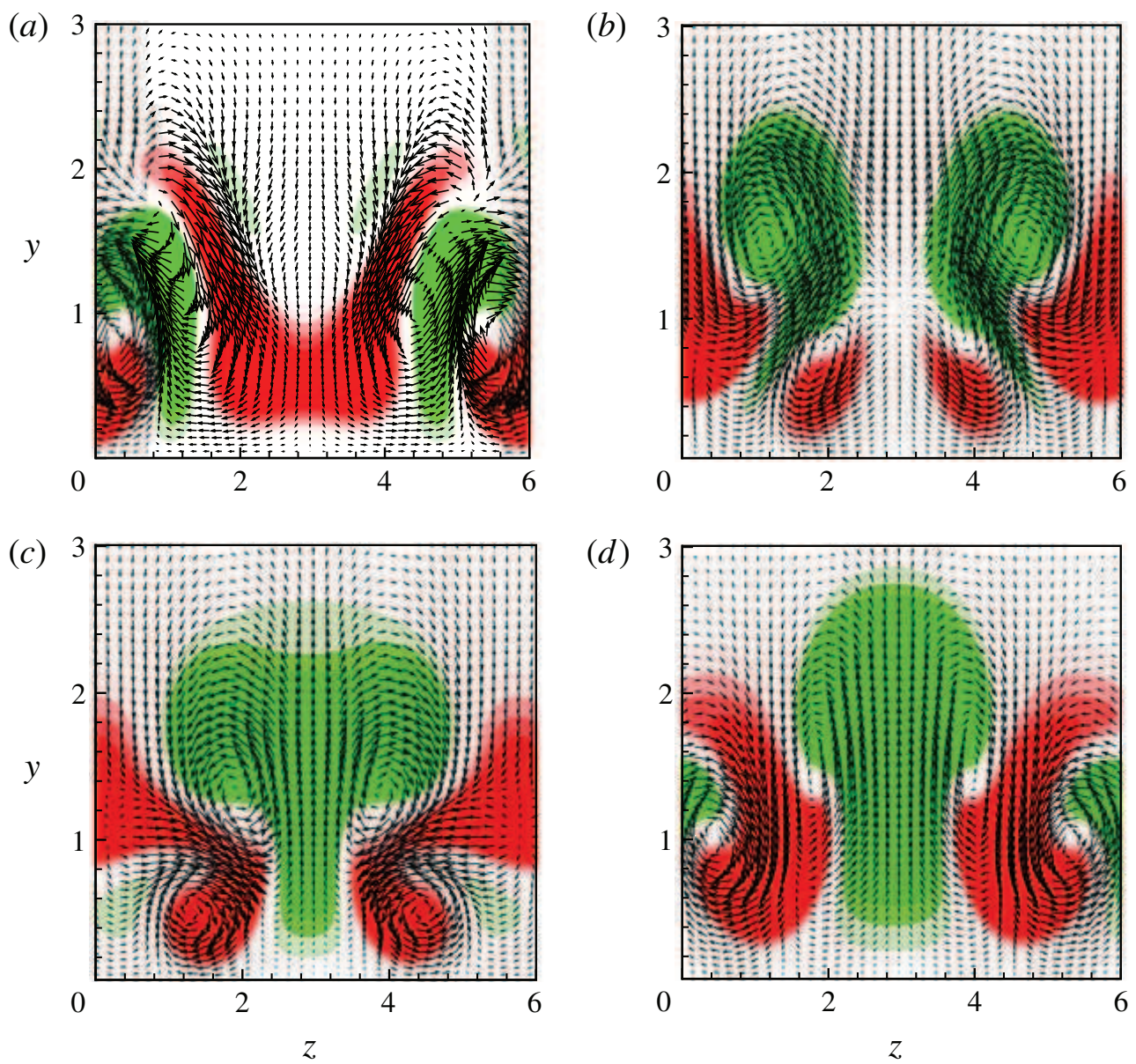

FIGURE 12. (Colour online) Contours and vectors of the velocity components of the optimal perturbation at $t=75$ obtained for $R e=610$ and $E_{0}=0.01$, on the planes $x=264(a)$, $x=268(b), x=270(c)$ and $x=274(d)$. Shaded contours indicate the streamwise velocity component of the perturbation (dark (red online) for positive values; light (green online) for negative values); vectors represent the wall-normal and the spanwise velocity component. Axes are not on the same scale.
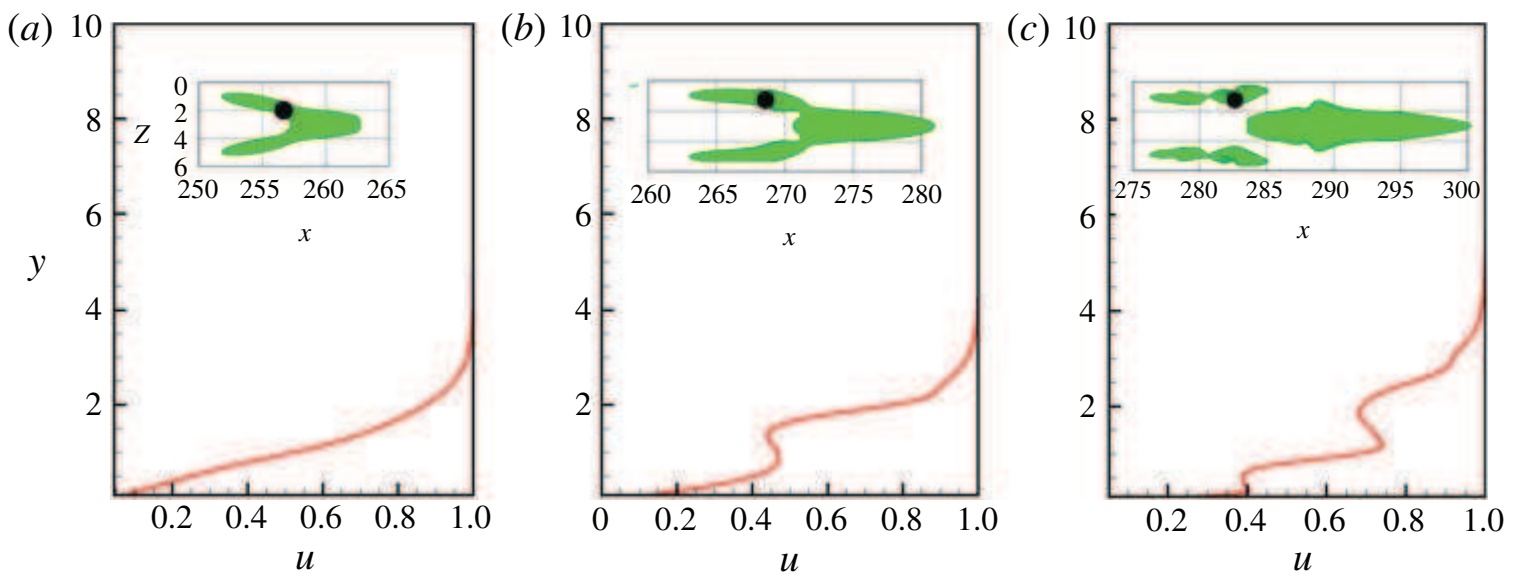

FIGURE 13. (Colour online) Profiles of the instantaneous streamwise velocity at three times, $t=50,75,100$ (from $a$ to $c$ ), along with isosurfaces of $u^{\prime}$ at each time. The black dots indicate the locations where the velocity profiles have been extracted. 


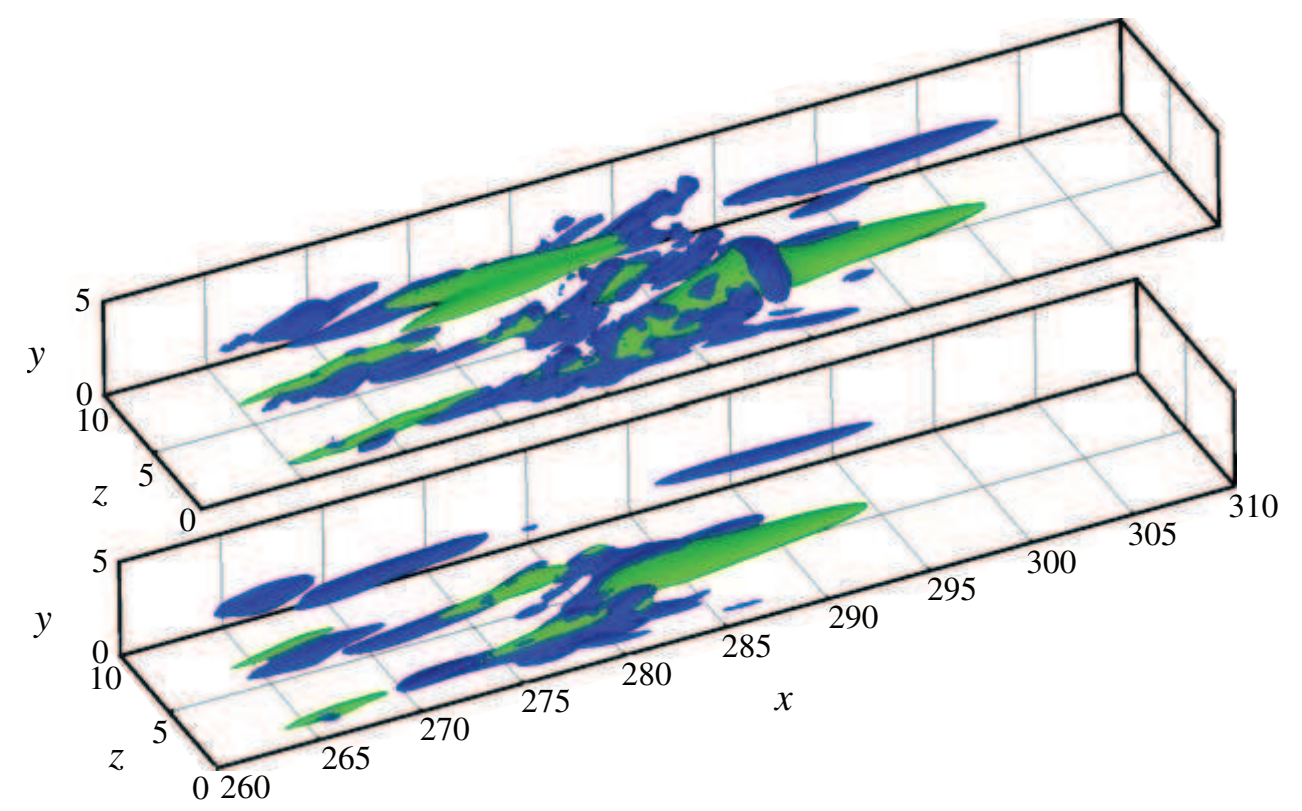

FIGURE 14. (Colour online) Isosurfaces of the negative streamwise component of the velocity perturbation (light (green online) for $u^{\prime}=-0.2$ ) and of the $Q$ criterion (dark (blue online) for $Q=2000$ ) obtained at $t=100$ by a complete DNS $(a)$ and by a DNS with $U$ multiplied by 0.9 at $t=111(b)$, both initialized by the minimal seed perturbation.

The correspondence of regions of streamwise and wall-normal velocity components of opposite sign and finite amplitude is an important feature of the minimal seed perturbation, since it strongly recalls the dynamics of a turbulent boundary-layer flow. In fact, it has been demonstrated that the mechanisms responsible for creating Reynolds shear stress in a boundary layer are mostly related to negative streamwise fluctuations being lifted away from the wall by positive wall-normal fluctuations (ejections), as well as to positive streamwise fluctuations approaching the wall (sweeps; see Corino \& Brodkey 1969; Willmarth \& Lu 1972). Such mechanisms have been linked to the burst phenomenon and, later, to the presence of hairpin vortices (Robinson 1991), so that they can be considered the kinematic basis of boundary-layer turbulence.

At this stage of the transition process, owing to the tilting of the initial vortices and to the dislocations induced by the interactions of finite amplitude perturbations, the downstream part of the vortex, which is the most distant from the wall, is convected downstream faster than the upstream part; since it experiences higher base flow velocity, the vortex is stretched in the streamwise direction. This is shown in the third frame of figure $11(t=65)$, where one can notice that the vortical structures and the low-momentum regions increase their intensity due to the vortex-stretching mechanism. Only after the two main vortices connect in their downstream part (third frame) does the main $\Lambda$-structure break up into two main legs connected by a vortex filament (fourth frame, $t=95$ ). The creation of a hairpin vortex characterizes the breakdown phase of the transition path initiated by the minimal seed (see also the movie included as supplementary material, available at journals.cambridge.org/flm). It is worth pointing out that the streamwise streaks begin to develop only after the creation of the hairpin head (see the fifth frame of figure 11 at $z \approx 2.8, z \approx 8.4$, and the first frame of figure 14). Thus, as already conjectured in Wu \& Moin (2009) and Cherubini et al. (2010a), the formation of long streamwise streaks appears to 
be a simple kinematic consequence of the presence of hairpin vortices. The same behaviour has been observed for all of the spanwise domain lengths considered here. In particular, to ascertain that the transition process involves the same spanwise length scales, the spanwise spacing of the streaks has been measured in the late stage of transition (at $t=200$ ) for three DNSs having $L_{z}=7, L_{z}=10.5$ and $L_{z}=15$, each one initialized by the minimal seed perturbations obtained using the corresponding computational domain (cf. figure 8). Normalizing the spanwise spacing with respect to the wall shear stress, one obtains $\lambda^{+} \approx 114.5$ for $L_{z}=15$ and $L_{z}=10.5$, which is close to the streak spacing commonly observed in turbulent boundary layers (e.g. Kline et al. 1967), whereas a smaller value, $\lambda^{+} \approx 98$, is found for $L_{z}=7$, due to the limited spanwise length of the computational box. A similar behaviour is recovered for all values of the Reynolds number, the initial energy, and the target time considered here, as soon as the nonlinearity threshold is overtaken.

\subsubsection{Analysis of the basic mechanisms of the transition process}

In the discussion above we have described the route leading the minimal seed perturbation to transition. Now, we are going to identify in a more quantitative way the basic mechanisms of this amplification process, as well as the role of the linear and nonlinear convective terms. Such an analysis is performed using DNSs, switching off or rescaling one by one the convective terms of the NS equations, and comparing the results with those obtained solving the complete NS equations. All of the computations discussed in this subsection have been performed initializing the flow by means of the minimal seed perturbation obtained for $R e=610, E_{0}=0.01$ and $T=75$.

Among the linear terms, we have found that those which mostly affect the route of the flow to transition are: (i) the term of convection of the streamwise component of the base flow by the wall-normal component of the perturbation, $v^{\prime} U_{y}$; (ii) the terms of convection of the perturbation by the streamwise component of the base flow, namely, $U u_{x}^{\prime}, U v_{x}^{\prime}$ and $U w_{x}^{\prime}$. Other linear convective terms have been found to play a small role due to the weak non-parallelism of the flow.

The term $v^{\prime} U_{y}$ is usually associated with the lift-up mechanism, in which slow/fast fluid is transported upwards/downwards in the boundary layer creating slow/fast streaks of streamwise perturbation and increasing the perturbation energy. In the present case, a mechanism similar to the lift-up is found, since, when such a term is switched off, no energy increase is found, and the maximum value of the streamwise velocity drops quickly (at $t=25, u_{\max }^{\prime}$ is 20 times lower than in the optimal case), inhibiting the triggering of nonlinear effects within the flow and leading to a fast relaminarization. This indicates that transition relies on the lift-up mechanism to allow an initial growth of the perturbation.

Concerning the terms of transport of the perturbation by the streamwise component of the base flow, they have a large effect on the flow dynamics, owing to the much larger value of $U$ with respect to $V, u^{\prime}, v^{\prime}$ and $w^{\prime}$. Thus, in such a case we have used a different diagnostic: three simulations have been performed in which the linear terms containing $U$ are multiplied by a factor $h$ smaller than one. The first and second frame of figure 14 show the disturbance structure at $t=100$ for the complete DNS, and at $t_{h}=t / h$ for a DNS with $h=0.9$, where the time has been scaled in order to take into account the different base-flow convection velocities. The most striking difference with respect to the complete case is the absence of the head of the hairpin vortex. In fact, reducing the convection of the perturbation by the base flow, the flow structures experience less stretching in the streamwise direction, and the $\Lambda$ vortices are not able to join on their downstream part to create the hairpin head. 

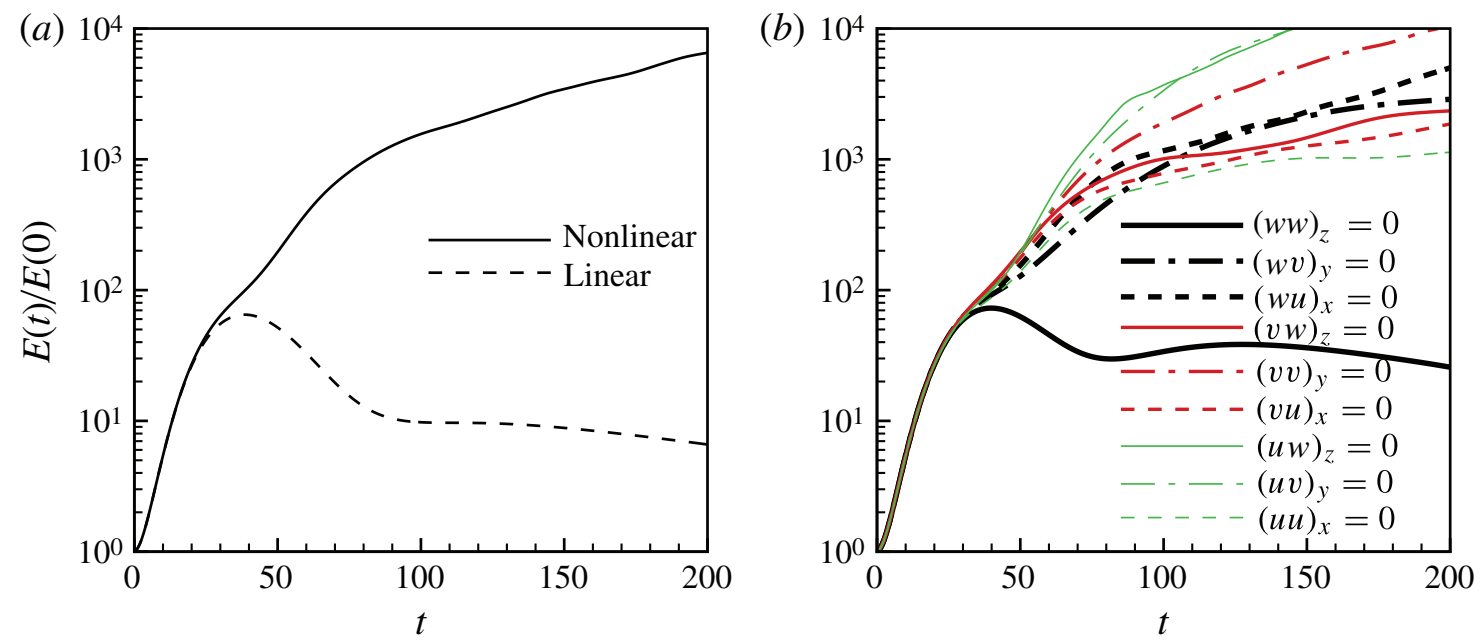

FIGURE 15. (Colour online) Evolution of the energy gain in time for several simulations initialized by the minimal seed perturbation: linear (dashed line) and nonlinear (solid line) DNS (a); DNSs performed by switching off one by one the nonlinear terms indicated within frame $(b)$.
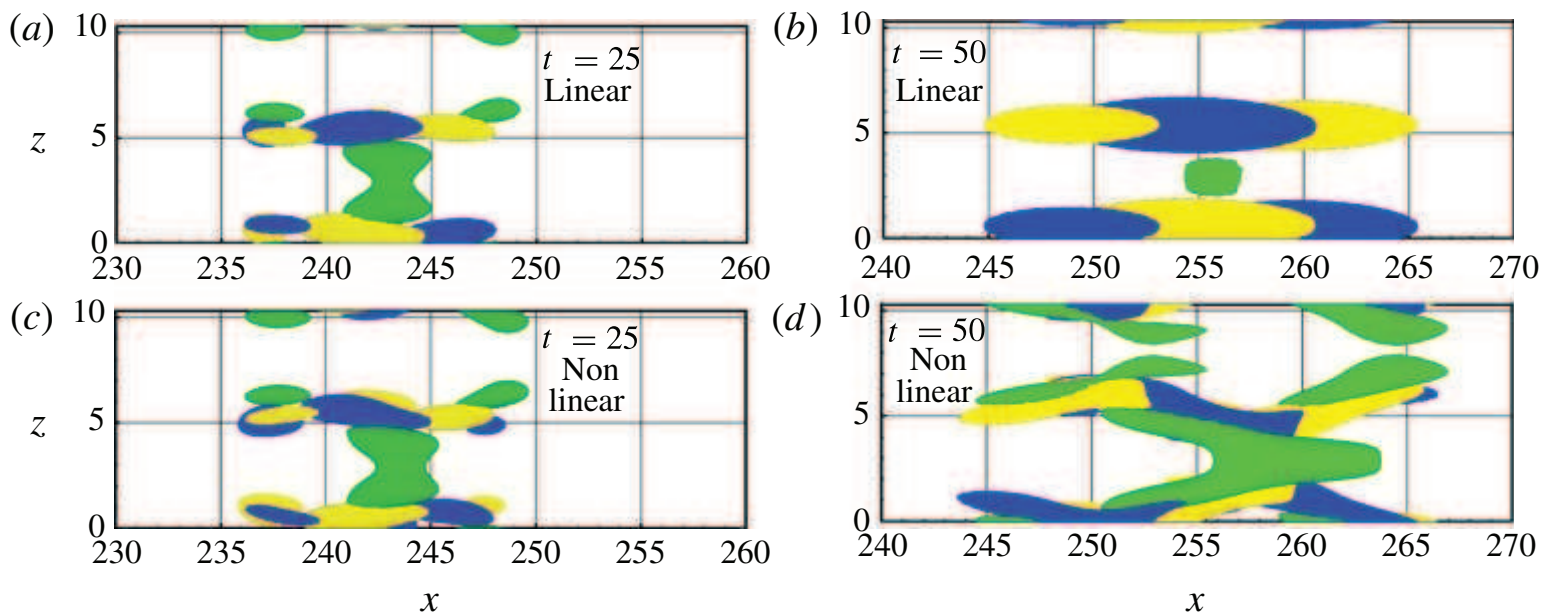

FIGURE 16. (Colour online) Isosurfaces of the perturbation obtained at $t=25(a, c)$ and $t=50(b, d)$, for a linear simulation $(a, b)$ and a complete nonlinear simulation $(c, d)$. The grey (green online) surfaces indicate the negative streamwise component of the perturbation velocity $\left(u^{\prime}=-0.05\right.$ for $t=25$ and $u^{\prime}=-0.08$ for $\left.t=50\right)$, the dark and light (blue and yellow online) surfaces indicate negative and positive streamwise vorticity, respectively $\left(\omega_{x}^{\prime}= \pm 0.15\right.$ for $t=25$ and $\omega_{x}^{\prime}= \pm 0.2$ for $\left.t=50\right)$.

The linear convective terms discussed above have an important role in transition. However, nonlinear terms are necessary to trigger turbulence and have a major effect in the development of the perturbation. This can be first verified by performing a simulation in which all of the nonlinear terms are switched off. Figure 15(a) shows the evolution of the energy gain in such a case (dashed line), compared to the complete DNS (solid line). One can notice the strong differences between the two curves for $t>25$, the first showing a decrease of the energy gain and a drop of the perturbation down to the laminar flow, the second showing a fast growth of the energy, followed by transition to turbulence. Thus, such curves confirm that, whereas for $t \leqslant 25$ the energy amplification is related to linear mechanisms, for $t>25$ it is nonlinearity which 
(a)

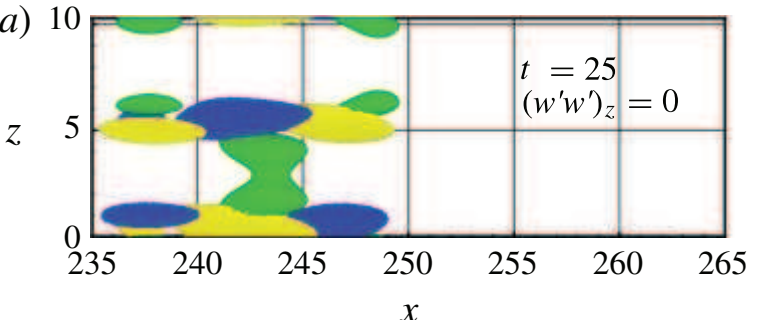

(b)

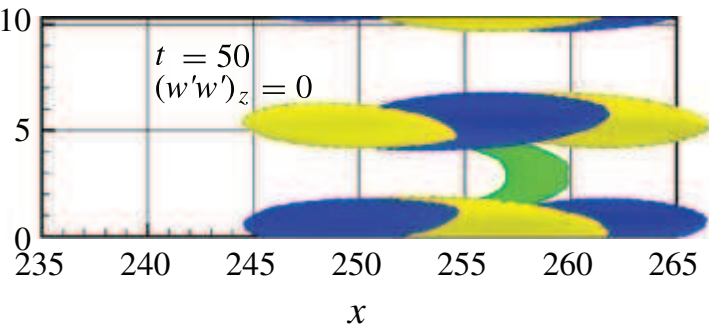

FIGURE 17. (Colour online) Isosurfaces of the perturbation obtained at $t=25(a)$ and $t=50$ (b) by a DNS with the term $\left(w^{\prime} w^{\prime}\right)_{z}$ switched off. The grey (green online) surfaces indicate the negative streamwise component of the perturbation velocity $\left(u^{\prime}=-0.05\right.$ for $t=25$ and $u^{\prime}=-0.08$ for $t=50$ ), the dark and light (blue and yellow online) surfaces indicate negative and positive streamwise vorticity, respectively $\left(\omega_{x}^{\prime}= \pm 0.15\right.$ for $t=25$ and $\omega_{x}^{\prime}= \pm 0.2$ for $t=50)$.

causes the disturbance energy to grow. The left frames of figure 16, providing the perturbations for the linear $(a, b)$ and the nonlinear $(c, d)$ case at $t=25$, show that the structure of the perturbations is almost identical in the two cases. On the other hand, at $t=50$ (figure $16 \mathrm{~b}, d$ ) strong modifications appear; in particular, the contribution of the nonlinear terms is fundamental to induce the lateral inclination of the vortices. In fact, in the absence of nonlinear terms, at such a time instant the vortices are aligned with the streamwise direction and the streamwise perturbation is much less intense compared with the nonlinear case. At larger times, both the vortices and the streaks continue to decrease their amplitude, leading to the relaminarization of the flow. This confirms that the lift-up mechanism alone is not capable of sustaining the perturbation, and indicates that the inclination of the vortices is a fundamental feature of the transition onset.

It is interesting to investigate which of the nonlinear terms are capable to sustain the inclination of the vortices against the mean flow. To this purpose, we performed nine numerical simulations in which the nonlinear terms (in conservative form) are switched off one by one. The corresponding energy growth curves are given in figure 15(b), and show that some terms provide a dissipative contribution, others contribute to production of $E(t)$. It is found that the most important term is that which couples the spanwise disturbance with its spanwise derivative: $\left(w^{\prime} w^{\prime}\right)_{z}$. Such a term plays a key role in sustaining transition, since it leads the flow to relaminarization when it is switched off. Comparing the dashed line in figure 15(a) with the thick solid line in figure $15(b)$, it appears that the energy gain curve is very similar to that previously found in the linear case; moreover, observing the perturbation structure at $t=25$ and $t=50$, shown in figure 17 (first and second frame, respectively), one can note that the effect obtained on the perturbation evolution switching off only such a term is very similar to that obtained switching off all of the nonlinear terms. Also in this case the vortices rapidly lose their initial inclination, inducing a decrease in the perturbation growth, although in such a case the streamwise disturbance decreases more slowly, and maintains for some time a $\Lambda$ shape. This behaviour confirms the fundamental role of the inclination of the vortices in the transition process studied here, and proves that the nonlinear term $\left(w^{\prime} w^{\prime}\right)_{z}$ is responsible to maintain such an inclination. This finding could be important for the design of efficient control strategies, since a relaminarization of the flow might be obtained by compensating the $\left(w^{\prime} w^{\prime}\right)_{z}$ coupling term by a careful use of sensors and actuators at the wall. 


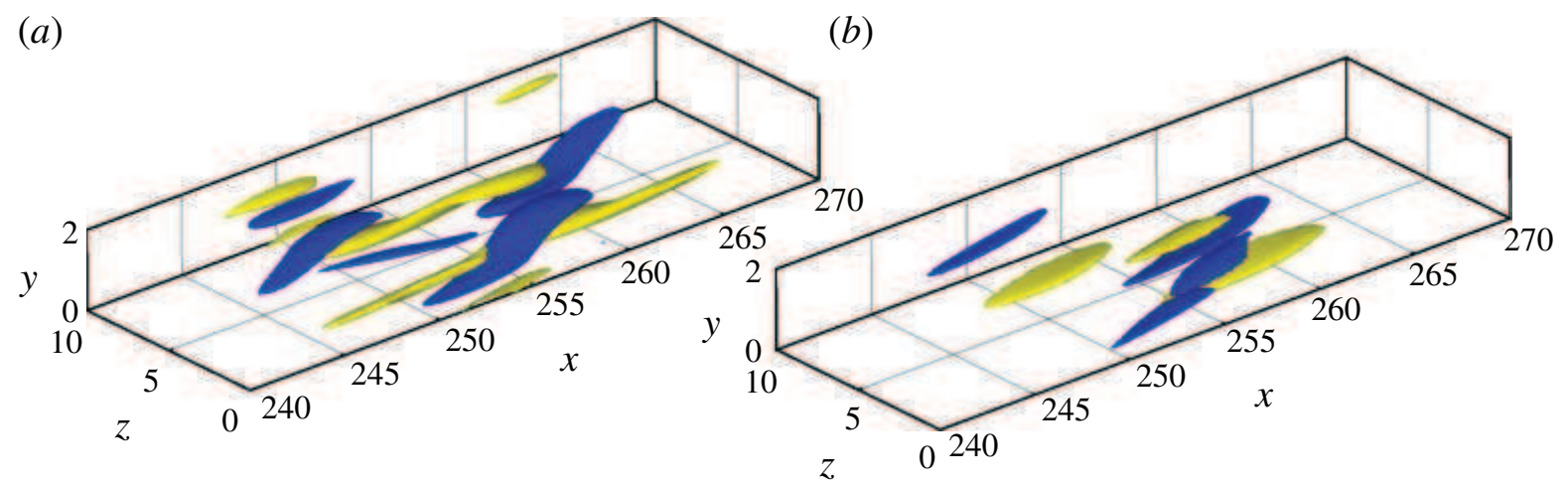

FIGURE 18. (Colour online) Isosurfaces of the perturbation obtained at $t=50$ by a complete DNS $(a)$ and by a DNS with the term $\left(w^{\prime} u^{\prime}\right)$ y switched off $(b)$. Light and dark (yellow and blue online) surfaces indicate positive and negative streamwise vorticity perturbation $\left(\omega_{x}^{\prime}= \pm 0.25\right)$.
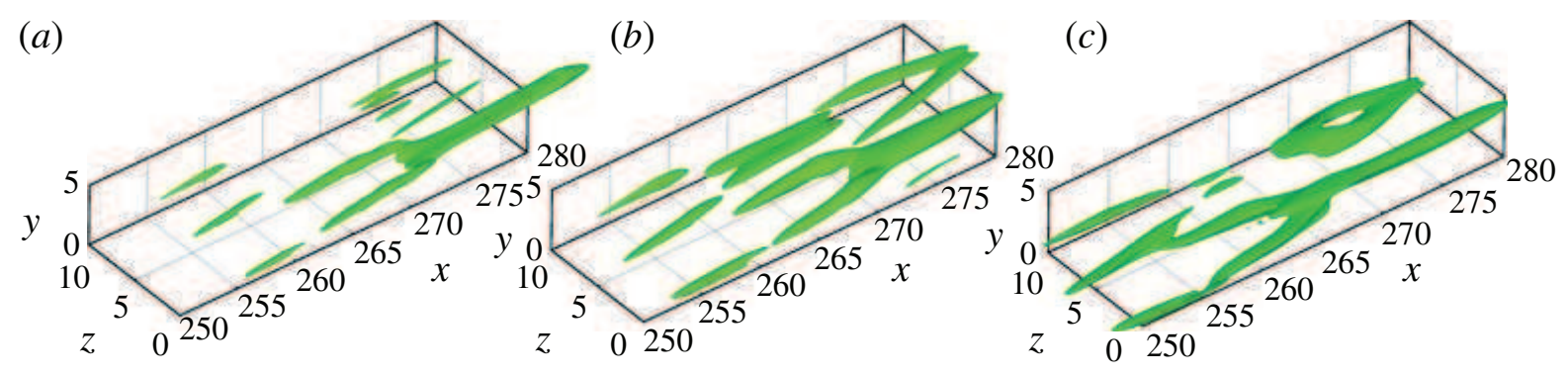

FIGURE 19. (Colour online) Isosurfaces of the perturbation (negative streamwise component of the velocity, $\left.u^{\prime}=-0.15\right)$ obtained at $t=75$ by a DNS having the term $\left(u^{\prime} u^{\prime}\right)_{x}$ switched off $(a)$, by a complete DNS $(b)$ and by a DNS having the term $\left(u^{\prime} v^{\prime}\right)_{y}$ switched off $(c)$.

Also other nonlinear terms have a significant effect on the development of the perturbation and its route to turbulence. A decrease of the energy gain around $t \approx 50$ is observed when the term $\left(w^{\prime} v^{\prime}\right)_{y}$ is switched off. Figure 18 shows the iso-surface of the streamwise vorticity at $t=50$ for a complete DNS $(a)$, and for a DNS with the $\left(w^{\prime} v^{\prime}\right)_{y}$ term switched off $(b)$. One can notice that, in the latter case, the vortices are weaker and lie closer to the wall, since the spanwise/wall-normal components of the velocity perturbation are not transported in the wall-normal direction. This inhibits the self-sustainment of the vortices, causing a substantial drop in the energy of the perturbations, eventually leading to the relaminarization of the flow (not shown in figure $15 b)$. A large impact is also produced by the $\left(u^{\prime} u^{\prime}\right)_{x}$ term, since, switching it off, the energy gain is one order of magnitude smaller with respect to the complete case at $t=250$ (see figure $15 b$ ). The amplification mechanisms linked to such a term play a role at slightly larger times than in the previous case. Figure 19(a) show that at $t=75$ large differences are present with respect to the complete case $(b)$. In particular, by switching off such a term, fewer dislocations in the plot of the streamwise component of the perturbation are found, and the creation of $\Lambda$ low-momentum structures is inhibited. As a result, the streamwise disturbance tends to be realigned with the streamwise direction, creating an array of elongated streaks. A similar effect is induced by switching off the term $\left(u^{\prime} v^{\prime}\right)_{y}$, although the underlying mechanism is slightly different, since this is a dissipative term (switching it off, an increase of the energy gain of 10 times is observed at $t=150$ ). The third frame of figure 19 shows 


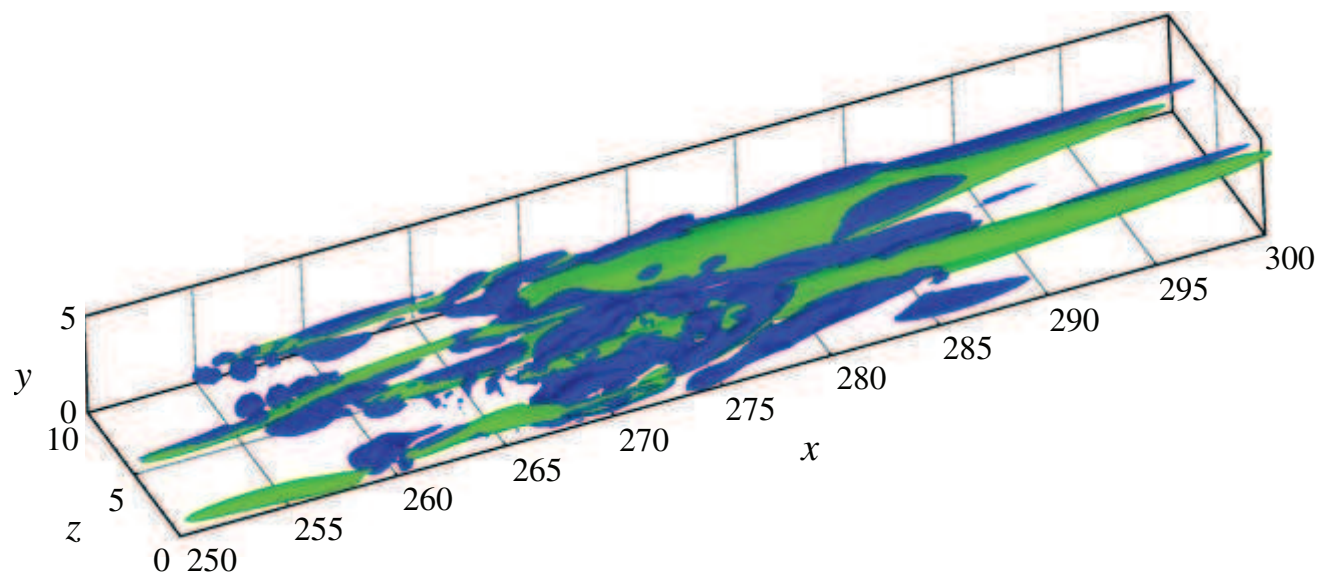

FIGURE 20. (Colour online) Isosurfaces of the negative streamwise component of the velocity perturbation (grey (green online) for $u^{\prime}=-0.2$ ) and $Q$ criterion (dark (blue online) for $Q=1500)$ obtained at $t=100$ by a DNS having the term $\left(u^{\prime} v^{\prime}\right)_{y}$ switched off.

that also in this case the resulting flow is populated by streaky structures localized close to the wall. In fact, the role of the term $\left(u^{\prime} v^{\prime}\right)_{y}$ in the nonlinear optimal transition scenario is to displace the streamwise finite amplitude perturbation up and down in the boundary layer, creating peaks of low momentum fluid at higher wall-normal positions, as well as peaks of high-momentum fluid close to the wall. Thus, when such a term is switched off, transition occurs following a route which does not privilege the creation of hairpin vortices. As shown in figure 20, at transition the flow is dominated by streamwise streaks and vortices, with remarkable differences with respect to the nonlinear optimal case (cf. figure 14a). In fact, transition appears to be due to the secondary instability of the streaks, which dominate the flow before breakdown. One can also notice that the flow structures observed in such a case closely recall those of one of the edge states identified by Cherubini et al. (2011), obtained by initializing the perturbation with a linear optimal disturbance. A similar behaviour is observed also when the dissipative term $\left(u^{\prime} w^{\prime}\right)_{z}$ is switched off, although the effect is now weaker.

Finally, it has been observed that switching off the term $\left(v^{\prime} u^{\prime}\right)_{x}$ inhibits the formation of the hairpin head, delaying the transition to turbulence. This is probably due to the fact that this term contributes to the spanwise vorticity, which is precisely the component which is needed in forming the head of the hairpin. The remaining terms cause only weak modifications of the perturbation structure, and do not affect the features of the transition scenario discussed so far.

\subsubsection{A conjecture about a disturbance regeneration cycle}

It is possible to summarize the above results by outlining a transition scenario based on the following successive steps.

(a) Tilting and amplification of the initial minimal seed by means of the linear Orr mechanism, resulting in a staggered array of elongated inclined vortices on the flanks of a low-streamwise-momentum region.

(b) Lift-up effect induced by the linear term $v^{\prime} U_{y}$, resulting in a further amplification of the streamwise disturbance alongside the vortical structures.

(c) Appearance of dislocations in the perturbation field induced by nonlinear coupling terms with (i) further streamwise tilting of the initial vortices due to the term $\left(w^{\prime} w^{\prime}\right)_{z}$, and sustainment of the vortical structures from the term $\left(w^{\prime} v^{\prime}\right)_{y}$; (ii) dislocations of the initial localized patches of finite amplitude streamwise 
(a)

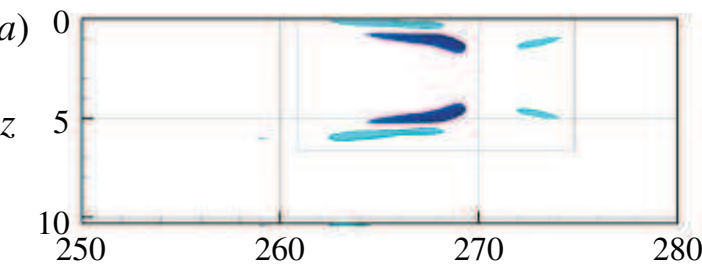

(c)

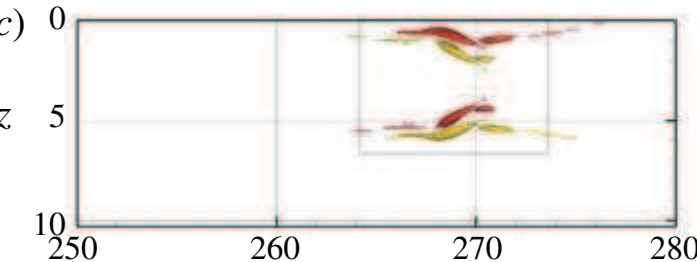

(e)

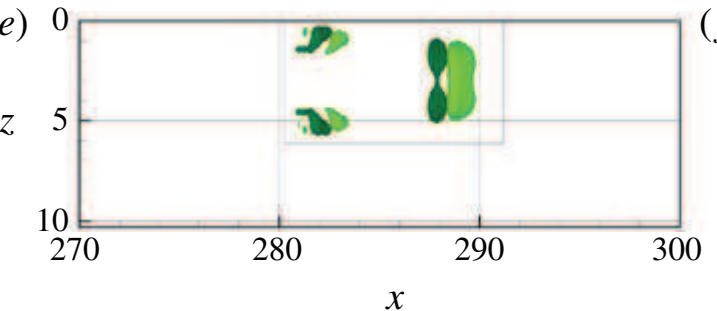

(b)

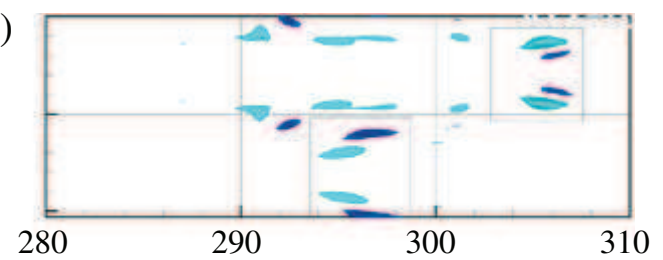

(d)

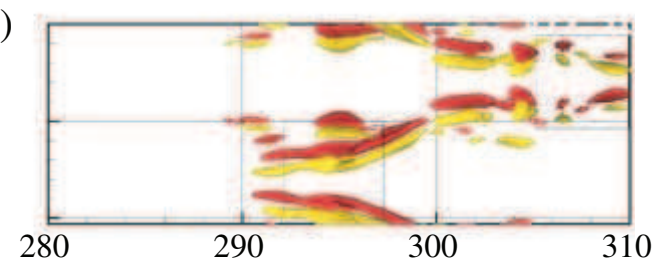

$(f)$

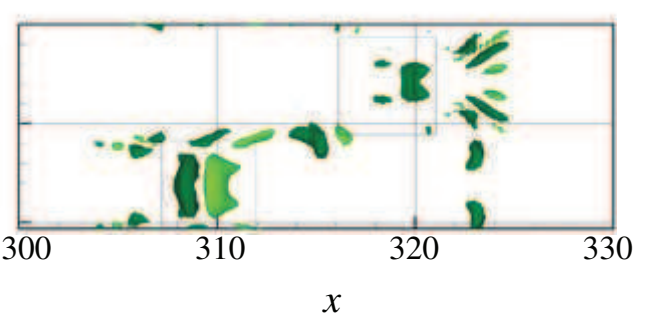

FIgURE 21. (Colour online) Isosurfaces of the lift-up term $v^{\prime} U_{y}= \pm 0.04$ at $t=75$ and $t=125$ ( $a$ and $b$, respectively); of the term $\left(w^{\prime} w^{\prime}\right)_{z}= \pm 0.025$ at $t=75$ and $t=125$ ( $c$ and $d$, respectively); of the $U v_{x}^{\prime}= \pm 0.13$ term at $t=100$ and $t=150$ ( $e$ and $f$, respectively).

disturbance associated with nonlinear terms such as $\left(u^{\prime} u^{\prime}\right)_{x}$ and $\left(u^{\prime} v^{\prime}\right)_{y}$, generating $\Lambda$-shaped structures of slow and fast fluids.

(d) Transport of the vortical structures by the mean flow, which stretches them in the streamwise direction, owing to the terms $U u_{x}^{\prime}, U v_{x}^{\prime}$ and $U w_{x}^{\prime}$; this causes an interaction of the vortices in their downstream part, resulting in the creation of $\Lambda$ vortices of finite amplitude.

(e) Redistribution of the vorticity due to nonlinear mixing (mostly related to the term $\left.\left(v^{\prime} u^{\prime}\right)_{x}\right)$, inducing the creation and the rise of a spanwise arch vortex; as a result, a hairpin is created.

(f) Release of smaller-scale vortices and hairpin structures from the main one (see also Adrian 2007).

It is noteworthy that such mechanisms can be observed at smaller scales for larger times. We have analysed at different times the regions of the flow where the main convective terms involved in the transition process are active, i.e. where they achieve a large value. For instance, observing the isosurfaces of the lift-up term $v^{\prime} U_{y}$ at time $t=75$, provided in the first frame of figure 21 , one can notice that such a term is active in the inclined elongated regions corresponding to the alternated inclined vortices characterizing the optimal disturbance at such time. Similar inclined elongated structures are observed at smaller scales for $t=125$ (see the boxed regions on the second frame of figure 21), meaning that the step $(b)$ of the transition process outlined above is repeated at smaller scales. At the same times, also the regions where the nonlinear term $\left(w^{\prime} w^{\prime}\right)_{z}$ is large show similar shapes and are characterized by smaller scales at larger times, as provided in the middle frames of figure 21 . Likewise for the $U v_{x}^{\prime}$ term (bottom frames of figure 21) at $t=100$ and $t=150$; it can be observed that such a term is active at $t=100$ in the zone where the main hairpin head is created (compare with the first frame of figure 14 at $x \approx 290$ ), and it is active again at $t=150$ 


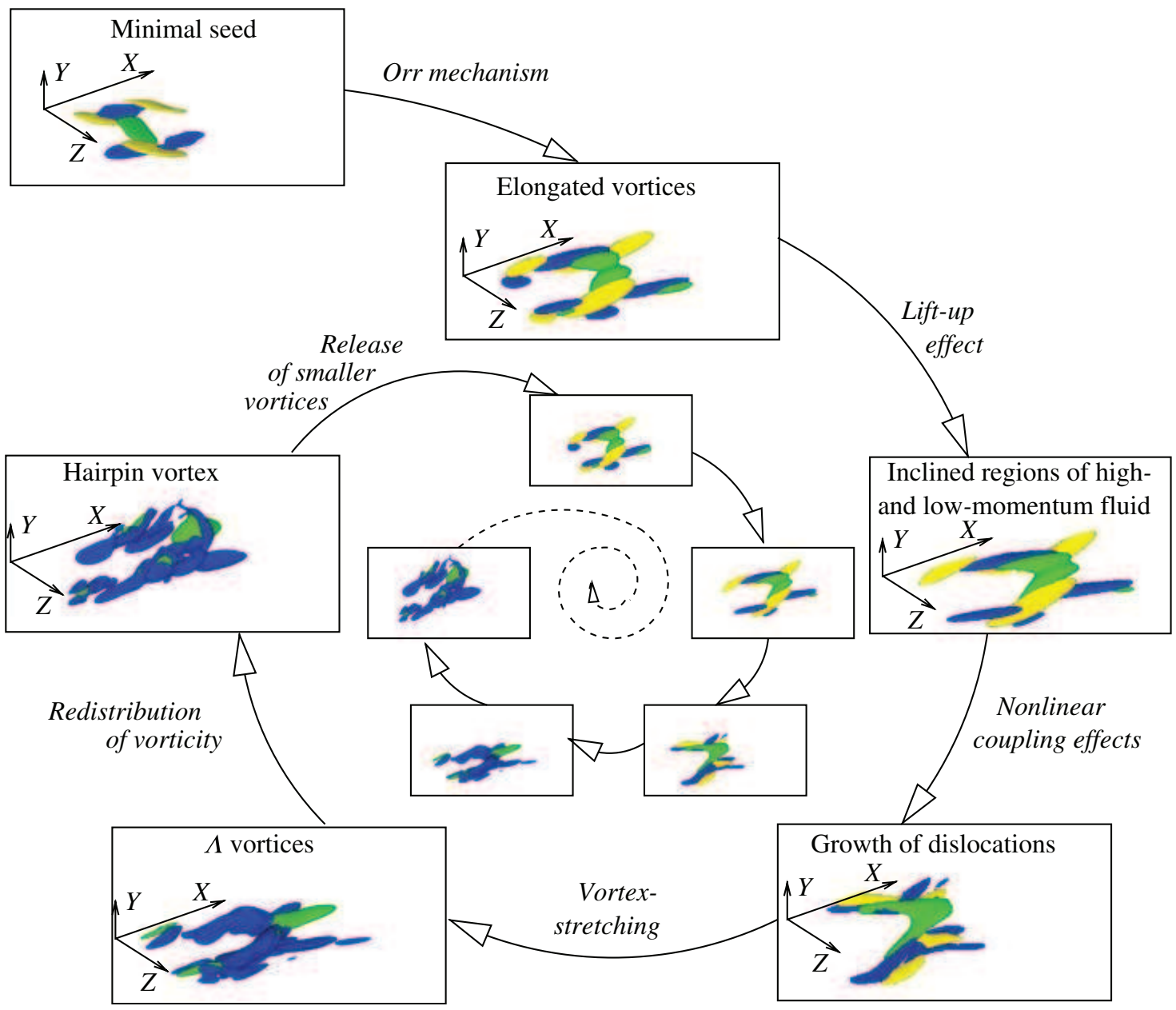

FIGURE 22. (Colour online) Sketch of the cycle of transition and disturbance-regeneration for the boundary-layer flow. The isosurfaces in the figures (following the cycle, up to 'Growth of dislocations') represent the negative streamwise component of the perturbation velocity (grey (green online)) and the positive and negative streamwise disturbance vorticity (light and dark (yellow and blue online), respectively). In the last two large frames, the green (grey) regions still represent patches of low streamwise perturbation velocity, while isosurfaces of the $Q$ criterion have been plotted in dark (blue online) to visualize regions of intense vorticity.

in the region corresponding to the head of a secondary hairpin (for $x \approx 310$ and $z \approx 8$ ), confirming the role of such a term in the creation of main and subsidiary hairpin vortices. Thus, it appears that also at larger times the mechanisms summarized in the previous subsection are at work, each playing a role in a similar way but at smaller scales.

These results suggest the existence of a regeneration cycle initiated by streamwiseinclined vortices, which turn into $\Lambda$ and hairpin structures and induce the release of smaller vortices from the main ones, allowing the cycle to be repeated over faster (smaller) time (space) scales. In fact, being advected downstream, the main hairpin vortex increases in size; then, from the main quasistreamwise vortices smaller vortical structures separate (see also Adrian 2007), probably due to inflectional instabilities linked to the appearance of defects (see figure 13 and related discussion). If such vortical structures have a small streamwise inclination (which is very likely), the optimal amplification cycle can be replicated, as sketched in figure 22. Figure 23 shows the disturbance wave packet at $t=200$, when transition to turbulence has occurred. The bottom frames show the local view of three regions of the flow 

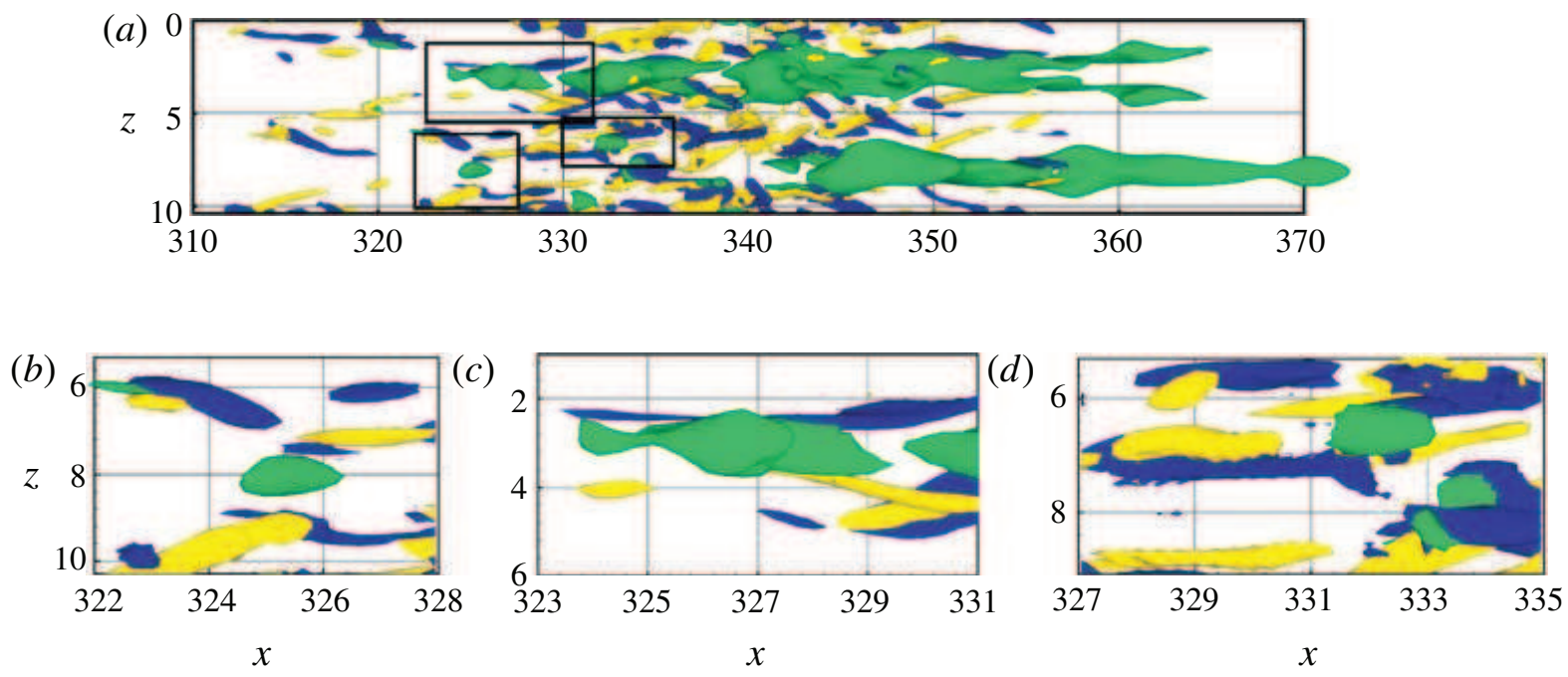

FIGURE 23. (Colour online) Isosurfaces of the streamwise component of the velocity (grey (green online) for $u^{\prime}=-0.25$ ) and streamwise vorticity perturbations (light and dark (yellow and blue online) for $\omega_{x}^{\prime}=0.6$ and $\left.\omega_{x}^{\prime}=-0.6\right)$, respectively. Panel $(a)$ shows the entire view of the wave packet, whereas panels $(b-d)$ provide the local view of the three regions of the flow marked by black rectangles on the top.

highlighting the presence of small-scale arrays of inclined vortices along with negative streamwise disturbance patches. These perturbations have a downstream inclination, closely recalling the elongated inclined vortices formed by the minimal seed in the second step of the transition scenario (see the second frame from the top in figure 22). Similar small-scale structures have been identified at different times, and for different initial perturbations (for $E_{0}$ and $T$ larger than the nonlinearity threshold), leading us to postulate the existence of a disturbance-regeneration cycle. Such a cycle is here initiated by the minimal seed perturbation, but its inception might be likely due also to free stream turbulence or other finite amplitude disturbances naturally occurring in a real flow (cf. for instance, the results in Wu \& Moin 2009). This cycle thus appears to be a good candidate to explain the late stages of transition to turbulence in a boundary-layer flow, based as it is on the regeneration of $\Lambda$ structures and hairpin vortices, preponderant features in this kind of flow (Adrian 2007; Wu \& Moin 2009), and grounded on well-recognizable amplification mechanisms.

\subsubsection{Comparison with other transition scenarios}

The transition scenario described here shows some features which recall the structures already observed in classical transition scenarios analysed in the literature. For example, the development of $\Lambda$ structures and hairpin vortices was also observed in the late stage of oblique transition (Berlin, Wiegel \& Henningson 1999). In this scenario, transition is induced by exciting oblique modes at the inflow, which grow and generate new modes through triad interactions. The streamwise-invariant modes which emerge grow much more rapidly than the others, due to the lift-up effect, inducing streaks which finally experience secondary instability and breakdown. On the other hand, in the case considered here the initial forcing is spatially localized, impulsively injected and characterized by a superposition of several modes. A comparison between the two scenarios can be performed by analysing the most amplified modes characterizing the time evolution of the nonlinear optimal perturbation. We have extracted such modes by means of a two-dimensional Fourier transform, identifying them by a pair of wavenumbers, $\alpha$ and $\beta$, in the streamwise 

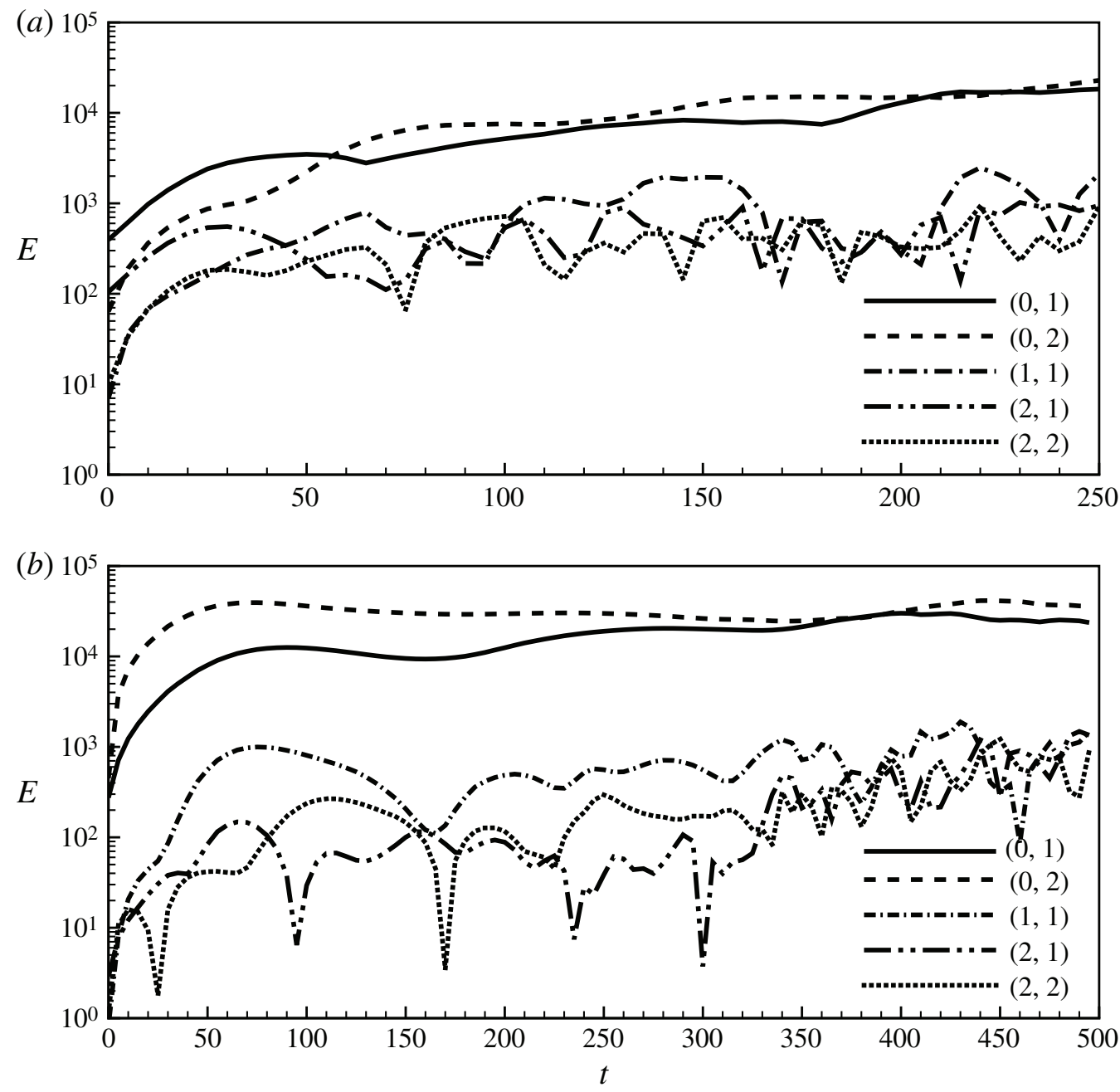

FIGURE 24. Two-dimensional Fourier transform providing the variation in time of the modes extracted by a DNS initialized by a nonlinear optimal perturbation with $E_{0}=0.01(a)$ and a linear optimal with $E_{0}=0.1(b)$. The modes are labelled as $(n, m)$.

and spanwise direction, respectively. The minimal seed with $E_{0}=0.01, T=75$ and $R e=610$ has been chosen to initialize the computations. Figure 24(a) shows the time history of the energy associated with such modes, labelled as $(n, m)$, where $n=\alpha /\left(2 \pi / L_{z}\right)$ and $m=\beta /\left(2 \pi / L_{z}\right)$, with $2 \pi / L_{z}$ the fundamental spanwise wavenumber defined by the characteristic length of the computational domain (the spanwise length $L_{z}$ has been chosen because $z$ is the only homogeneous direction). It is possible to compare this time history with that emerging from the oblique-transition scenario, for instance, that provided in figure 3 of Berlin, Lundbladh \& Henningson (1994), in which the computations where performed at $R e=400$ and for an initial amplitude of the perturbation $A=0.01$ (thus close to the parameters used here). Unlike the case of the oblique-transition scenario, the increase of the $(0,2)$ mode is not very rapid (for instance, the mode increases by two orders of magnitude in 100 time units, instead of 30 time units as in the oblique scenario). Indeed, this mode appears to grow at a rate which is similar to that of the other modes, especially mode $(0,1)$, whose growth is not observed in the oblique scenario. When transition occurs $(t \approx 250)$, such zero-streamwise-wavenumber modes attain energy values which are higher than those associated with the non-zero streamwise wavenumber components. It is worth specifying that most of the energy of such modes is not linked to the 
development of streaks or streamwise invariant perturbations, but it is due to the fact that, the flow not being periodic in the streamwise direction, the disturbances have mean value in the streamwise direction different from zero. Moreover, mode $(1,1)$ has a low energy level at the initial time, the most energetic modes being $(0,1)$, $(0,2)$ and $(2,1)$. The first two modes account for the fact that the initial perturbation has a mean value in the streamwise direction which is non-zero, whereas the third mode accounts for the spatial localization of the disturbance. Mode $(2,1)$ does not experience a large growth in time, unlike the other modes, which increase their energy due to the combined effect of lift-up, nonlinear mixing and vortex regeneration. This behaviour is remarkably different from the oblique transition scenario. Since the amplitude of the perturbation is very close to that used in Berlin et al. (1994), this behaviour is mostly due to its particular shape. This can be easily shown by comparing such a transition path to that induced by a linear optimal perturbation. Thus, to initialize the computations, a linear optimal perturbation with initial energy ten times larger than that used in the nonlinear case has been chosen $\left(E_{0}=0.1\right)$, in order to reach transition. The time history of the energy associated with the most amplified modes characterizing the linear optimal perturbation is provided in figure $24(b)$. It can be observed that, in the linear case, a very rapid increase of the $(0,2)$ mode is observed, due to the streaks generation induced by the initial streamwise vortices, followed by a saturation. A similar behaviour is displayed by mode $(0,1)$, although a slower increase is observed. At the same time, the mode $(1,1)$ experiences an initial increase, and then oscillates around a constant value. Modes $(2,1)$ and $(2,2)$ experience a first increase at short times, due to nonlinear effects induced by the growth of modes $(1,1)$ and $(0,2)$, and a second increase at $t \approx 350$, due to the onset of a secondary instability of the streaks. Most of these features have been observed in previous works on the oblique transition scenario (see figure 3 in Berlin et al. 1994); this means that the linear optimal disturbance reaches turbulence by following a path which is similar to the oblique case, which appears to be the most efficient in triggering transition for parallel flows in small domains (Reddy et al. 1998; Duguet et al. 2010). On the other hand, in the case of a nonlinear optimal disturbance, streamwise streaks are not observed until transition is initiated, so that the mechanism of secondary instability of the streamwise streaks is skipped. Instead, nonlinear effects cause an increase of all of the modal components of the perturbation at short times, explaining the differences between such a scenario and the oblique case.

These differences result in a different spreading of the disturbance, which can be visualized in figure 25 , providing the space-time diagram of the streamwise component of the velocity measured at $y=1, z=5$, for a nonlinear and a linear optimal initial conditions. It is clearly shown that in the nonlinear optimal case (figure 25a) the disturbance has a larger spreading rate in space, both at the leading and at the trailing edge of the wave packet. In particular, the leading edge of the wave packet is convected downstream at the velocity $U_{\text {lead }} \approx 0.9 U_{\infty}$, whereas the trailing edge is advected at $U_{\text {trail }} \approx 0.5 U_{\infty}$ (the presence of the main hairpin vortex at the leading edge explaining the large value of $U_{\text {lead }}$ ). Such values of velocity are very close to the advection velocities of the edges of a turbulent spot reported in the literature (Singer 1996). Moreover, the spreading rate at small times appears to be the same as that of the turbulent spot, which is established in the flow at $t \approx 250$. On the other hand, for a linear initialization the spatial spreading is much smaller in the first phase of the oblique transition, when streamwise streaks are generated (clearly visible in figure $25 b)$, and it only increases when a turbulent spot is established $(t \approx 450)$. 

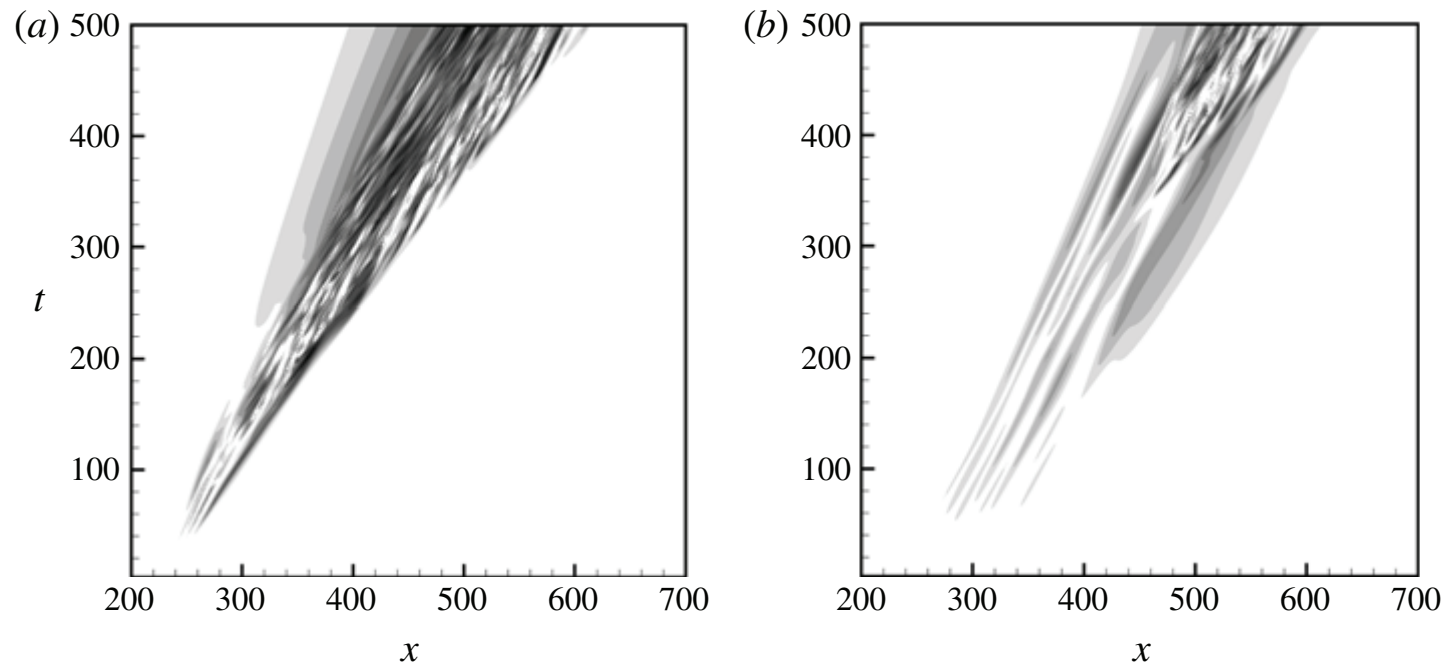

FIGURE 25. Space-time diagram of the streamwise component of the velocity perturbation extracted at $y=1, z=5$ by a DNS initialized by a nonlinear perturbation with $E_{0}=0.01$ (a) and a linear optimal disturbance with $E_{0}=0.1(b)$. The contours show 10 values of velocity from $\left|u^{\prime}\right|=0.05$ to $\left|u^{\prime}\right|=0.5$.

Such results thus confirm that the nonlinear optimal perturbation is able to initialize turbulence very rapidly, with a fast spreading in space and time, until a turbulent spot forms.

\section{Summary}

We have used a variational procedure to identify nonlinear optimal disturbances in a boundary-layer flow developing over a flat plate, defined as those initial perturbations yielding the largest energy growth at a given target time $T$, for a given Reynolds number $R e$. The analysis has been performed for two values of $R e$ and several values of $T$ and initial energy density $E_{0}$. For all values of $R e$ and $T$ a threshold value of $E_{0}$ exists, called the nonlinearity threshold, from which remarkable modifications are observed with respect to the linear case, in particular for the value of the nonlinear optimal energy at $T$. The nonlinearity threshold decreases when $R e$ and/or $T$ increase. Moreover, for values of the initial energy larger than the threshold, the optimal perturbation changes slightly and is characterized by a fundamental structure, called here the minimal seed, which has been analysed in detail. The same minimal seed structure, characterized by similar size and amplitude, is recovered also for different domain lengths, target times and Reynolds numbers, demonstrating that this is a robust feature of the considered flow. Unlike the linear optimal perturbations, the minimal seed contains vortices inclined in the streamwise direction surrounding a patch of intense streamwise disturbance velocity. These features induce a more rapid energy growth with respect to the linear case, due to the streamwise modulation of the momentum and to the significant distortion of the base flow profile.

DNSs have then been employed to study the mechanism of transition to turbulence when the flow is initialized using the minimal seed. It has been shown that, for the same value of the initial energy, the nonlinear optimal perturbation is able to lead the flow to turbulence whereas the linear optimal perturbation is not. The nonlinear optimal perturbation has been found to trigger and spread out turbulence very efficiently, its spreading rate in space and time being very close to that of a turbulent spot. The present transition scenario is quite different from the oblique- 
transition scenario, as well as from other classical scenarios, and this leads us to outline a new path of turbulent transition in the boundary layer, based on the fast growth and nonlinear evolution of the initial minimal seed. The mechanisms which characterize such a route to turbulence has been analysed performing DNSs, initialized by the minimal seed perturbation, in which the convective terms have been switched off or rescaled one by one to infer their effect. We have thus found that the transition scenario is basically composed of the following steps.

(a) Tilting and amplification of the initial minimal seed by means of the Orr mechanism, resulting in a staggered array of elongated inclined vortices on the flanks of a low-streamwise-momentum region.

(b) Transport of the base flow momentum by the disturbance (lift-up) along the inclined vortices, resulting in a further amplification of the streamwise disturbance.

(c) Dislocation of the initial localized patches of finite amplitude disturbance due to the self-interactions of the perturbation field, generating $\Lambda$-shaped structures of slow and fast fluids, and sustaining the initial inclination of the vortices.

(d) Transport of the vortical structures by the mean flow, which stretches them in the streamwise direction, resulting in the creation of $\Lambda$ vortices of finite amplitude.

(e) Redistribution of the vorticity due to nonlinear mixing, inducing the creation and the lift-up of a spanwise vorticity zone (the arch vortex) connecting two neighbouring vortex structures, constituting a main hairpin vortex.

( $f$ ) Release of smaller-scale vortices from the main hairpin because of inflectional instabilities related to the base flow modifications.

In particular, observing the activation in time of the convective terms connected to the amplification mechanisms summarized above, we have found that when the main hairpin releases smaller-scale vortical structures, new, localized inclined vortices are generated, allowing the cycle to repeat over faster (smaller) time (space) scales. Such a cycle is here initiated by the minimal seed perturbation, but its inception can also be due to free stream turbulence or other finite amplitude disturbances occurring in a flow. Future work will aim at evaluating the effects of such initial forcing on the nonlinear optimal disturbance, trying to include the receptivity mechanisms in the optimization procedure.

\section{Acknowledgements}

Some computations have been performed on the Power 6 of the IDRIS, France. The authors would like to thank R. Verzicco for providing the DNS code.

Supplementary movies are available at journals.cambridge.org/flm.

\section{Appendix. Nonlinear optimizations}

The nonlinear optimization has been carried out using a Lagrange multiplier technique, similar to that used by Zuccher et al. (2004) for a boundary-layer flow described by the (spatially parabolic) boundary-layer equations, and by Pringle \& Kerswell (2010) for a pipe flow. This technique consists of seeking extrema of the augmented functional $\mathscr{L}$ with respect to every independent variable. Such a functional is written as

$$
\mathscr{L}=G-\int_{0}^{T} \int_{V} a\left(u_{x}^{\prime}+v_{y}^{\prime}+w_{z}^{\prime}\right) \mathrm{d} V \mathrm{~d} t-\int_{0}^{T} \int_{V} b\left[u_{t}^{\prime}+v^{\prime} U_{y}+V u_{y}^{\prime}+\left(u^{\prime} U+u^{\prime} u^{\prime}\right)_{x}\right.
$$




$$
\begin{aligned}
& \left.+\left(u^{\prime} v^{\prime}\right)_{y}+\left(u^{\prime} w^{\prime}\right)_{z}+p_{x}^{\prime}-\frac{u_{x x}^{\prime}+u_{y y}^{\prime}+u_{z z}^{\prime}}{R e}\right] \mathrm{d} V \mathrm{~d} t \\
& -\int_{0}^{T} \int_{V} c\left[v_{t}^{\prime}+U v_{x}^{\prime}+u^{\prime} V_{x}+\left(u^{\prime} v^{\prime}\right)_{x}+\left(v^{\prime} v^{\prime}+v^{\prime} V\right)_{y}+\left(v^{\prime} w^{\prime}\right)_{z}\right. \\
& \left.+p_{y}^{\prime}-\frac{v_{x x}^{\prime}+v_{y y}^{\prime}+v_{z z}^{\prime}}{R e}\right] \mathrm{d} V \mathrm{~d} t \\
& -\int_{0}^{T} \int_{V} d\left[w_{t}^{\prime}+U w_{x}^{\prime}+V w_{y}^{\prime}+\left(w^{\prime} u^{\prime}\right)_{x}+\left(w^{\prime} v^{\prime}\right)_{y}+\left(w^{\prime} w^{\prime}\right)_{z}\right. \\
& \left.+p_{z}^{\prime}-\frac{w_{x x}^{\prime}+w_{y y}^{\prime}+w_{z z}^{\prime}}{\operatorname{Re}}\right] \mathrm{d} V \mathrm{~d} t-\lambda_{0}\left[\frac{E_{0}}{E(0)}-1\right]
\end{aligned}
$$

where the NS equations (direct problem) and the value of the energy at $t=0, E_{0}$, have been imposed as constraints, and $a, b, c, d, \lambda_{0}$ are Lagrange multipliers. Integrating by parts and setting to zero the first variation of $\mathscr{L}$ with respect to $u^{\prime}, v^{\prime}, w^{\prime}, p^{\prime}$ leads to the adjoint equations:

$$
\begin{gathered}
-b_{t}=2 b_{x} u^{\prime}+b_{y} v^{\prime}+b_{z} w^{\prime}+b_{x} U+(b V)_{y}-c V_{x}+a_{x}+\frac{b_{x x}}{R e}+\frac{b_{y y}}{R e}+\frac{b_{z z}}{R e} \\
-c_{t}=c_{x} u^{\prime}+2 c_{y} v^{\prime}+c_{z} w^{\prime}+(c U)_{x}+c_{y} V-b U_{y}+a_{y}+\frac{c_{x x}}{R e}+\frac{c_{y y}}{R e}+\frac{c_{z z}}{R e} \\
-d_{t}=d_{x} u^{\prime}+d_{y} v^{\prime}+2 d_{z} w^{\prime}+\mathrm{d} U_{x}+(\mathrm{d} V)_{y}+a_{z}+\frac{d_{x x}}{R e}+\frac{d_{y y}}{R e}+\frac{d_{z z}}{R e} \\
0=b_{x}+c_{y}+d_{z}
\end{gathered}
$$

where $\boldsymbol{q}^{\dagger}=(a, b, c, d)^{\mathrm{T}}$ is defined as the adjoint vector. The adjoint equations are linked to the direct equations by the presence of direct variables in the advection terms. By using the boundary conditions of the direct problem, we obtain

$$
\begin{array}{cll}
b=0, \quad c=0, & d=0, & \text { for } y=0 \text { and } y=L_{y}, \\
b=0, \quad c=0, & d=0, & \text { for } x=x_{\text {in }} \text { and } x=x_{\text {out }}
\end{array}
$$

moreover, the compatibility conditions (Zuccher et al. 2004) are

$$
\frac{2 u^{\prime}}{E_{0}}-b=0, \quad \frac{2 v^{\prime}}{E_{0}}-c=0, \quad \frac{2 w^{\prime}}{E_{0}}-d=0, \quad \text { for } t=T .
$$

The direct and adjoint equations are parabolic in the forward and backward time direction, respectively, so that they can be solved by a coupled iterative approach, similar to that used by Cherubini et al. (2010b). By solving the direct and adjoint equations at each step of the iterative procedure, the first variation of the augmented functional with respect to $\boldsymbol{q}$ and $\boldsymbol{q}^{\dagger}$ is set to zero. Moreover, the gradient of $\mathscr{L}$ with respect to the initial state $\boldsymbol{q}_{0}$ has to vanish within a reasonable number of iterations. Different methods can be used to achieve this goal. For instance, one can set to zero the gradient of $\mathscr{L}$ with respect to the initial state at each step of the iteration, achieving directly an estimate of the initial condition for the next step of the procedure (an 'optimality' condition, currently used in linear optimization problems). This strategy is effective in a linear framework in which the optimization procedure is guaranteed to converge to the global optimum (see Corbett \& Bottaro 2000 and Cherubini et al. 2010b), but it does not guarantee efficient convergence in a nonlinear problem such as that considered here. Therefore, in order to achieve convergence 
efficiently, a conjugate gradient algorithm is used, similar to that employed in Marquet et al. (2008). The initial state is updated in the steepest ascent direction, denoted as $\nabla_{q_{0}} \mathscr{L}$, namely:

$$
\begin{aligned}
& \frac{\partial \mathscr{L}}{\partial u_{0}^{\prime}}=-2 u_{0}^{\prime} \frac{\left(E(T)-\lambda_{0} E_{0}\right)}{E(0)}+b(0), \\
& \frac{\partial \mathscr{L}}{\partial v_{0}^{\prime}}=-2 v_{0}^{\prime} \frac{\left(E(T)-\lambda_{0} E_{0}\right)}{E(0)}+c(0), \\
& \frac{\partial \mathscr{L}}{\partial w_{0}^{\prime}}=-2{w^{\prime}}_{0} \frac{\left(E(T)-\lambda_{0} E_{0}\right)}{E(0)}+d(0),
\end{aligned}
$$

with an adjustable step length $\alpha$, so that $\boldsymbol{q}_{0}^{(n+1)}=\boldsymbol{q}_{0}^{n}+\alpha^{n} \nabla_{q_{0}} \mathscr{L}^{n}$. After the first iteration in the steepest ascent direction (with $\alpha$ sufficiently large for the solution to be significantly modified), the successive steps are taken along a conjugate direction, $\Lambda \boldsymbol{q}_{0}$, which is computed on the basis of the gradient at two consecutive iterations according to $\Lambda \boldsymbol{q}_{0}^{(n+1)}=\nabla_{q_{0}} \mathscr{L}^{(n+1)}+\beta^{(n+1)} \Lambda \boldsymbol{q}_{0}^{n}$. In the present work the value of the parameter $\beta^{(n+1)}$ is computed by means of the Polak-Ribière formula (Polak \& Ribière 1969),

$$
\beta^{(n+1)}=\frac{\left(\Delta \boldsymbol{q}_{0}^{(n+1)}\right)^{\mathrm{T}}\left(\Delta \boldsymbol{q}_{0}^{(n+1)}-\Delta \boldsymbol{q}_{0}^{n}\right)}{\left(\Delta \boldsymbol{q}_{0}^{n}\right)^{\mathrm{T}} \Delta \boldsymbol{q}_{0}^{n}} .
$$

Periodically, this value should be reset to zero in order to avoid conjugacy loss (which corresponds to updating the solution in the steepest ascent direction). The step length $\alpha$ has been chosen carefully in order to ensure convergence to the optimal value, as described below, with values in the range $\left[0.001 / E_{0}, 0.1 / E_{0}\right]$.

The optimization procedure for a chosen target time $T$ can be summarized as follows.

(a) An initial guess is made for the initial condition, $\boldsymbol{q}_{0}$, with associated initial energy $E_{0}$ (in most of the computations, the perturbation obtained by a linear optimization has been used as initial guess).

(b) The direct problem is integrated up to $t=T$.

(c) The adjoint variables, $b(T), c(T), d(T)$, are provided by the compatibility conditions (A 7).

(d) The adjoint problem (A2)-(A 5) is integrated backward in time from $t=T$ to $t=0$.

(e) At $t=0$, the initial direct state is updated in the direction of the conjugate gradient with step length $\alpha$ and $\beta$ computed according to the Polak-Ribière formula ( $\beta=0$ is imposed at the first iteration).

$(f)$ The objective function $E(T)$ is evaluated:

(i) if its increase between two successive iterations is smaller than a chosen threshold, $e$, the loop is stopped, otherwise the procedure is continued from step $(b)$;

(ii) if a decrease of the objective function is found, the value of $\alpha$ is halved, and the value of $\beta$ is set to zero.

Each iteration requires the integration of the three-dimensional NS and adjoint equations forward and backward in time up to the target time; moreover, owing to the presence of the direct variables in the advection terms of the adjoint equations, the 
whole direct-variable field needs to be stored at each time step, requiring a remarkable storage capacity. Considering that $\sim 200$ iterations are needed to achieve convergence within machine accuracy, the computational effort required to optimize the solution for a single set of the independent parameters $\left(T, R e\right.$ and $\left.E_{0}\right)$ is very high. To keep the computational cost affordable, the optimizations have been performed up to a target time $T=125$ and a threshold value equal to $e=10^{-6}$ has been chosen, after having verified that it produces an error on the value of $E(T)$ smaller than $0.5 \%$ (a more detailed convergence study is provided in Cherubini et al. 2010a).

\section{REFERENCES}

Adrian, R. J. 2007 Hairpin vortex organization in wall turbulence. Phys. Fluids 19, 041301.

Berlin, S., Lundbladh, A. \& Henningson, D. S. 1994 Spatial simulations of oblique transition in a boundary layer. Phys. Fluids 6, 1-3, 1949.

Berlin, S., Wiegel, M. \& Henningson, D. S. 1999 Numerical and experimental investigations of oblique boundary layer transition. J. Fluid Mech. 393, 23-57.

Biau, D. \& Bottaro, A. 2009 An optimal path to transition in a duct. Phil. Trans. R. Soc. A 367, 529-544.

Bottaro, A. 1990 Note on open boundary conditions for elliptic flows. Numer. Heat Transfer B 18, 243-256.

Bottaro, A. 1993 On longitudinal vortices in curved channel flow. J. Fluid Mech. 251, 627-660.

Brandt, L., Schlatter, P. \& Henningson, D. S. 2004 Transition in a boundary layers subject to free stream turbulence. J. Fluid Mech. 517, 167-198.

Cherubini, S., De Palma, P., Robinet, J.-Ch. \& Bottaro, A. $2010 a$ Rapid path to transition via nonlinear localized optimal perturbations. Phys. Rev. E 82, 066302.

Cherubini, S., De Palma, P., Robinet, J.-Ch. \& Bottaro, A. 2011 Edge states in a boundary layer. Phys. Fluids 23, 051705.

Cherubini, S., Robinet, J.-Ch., Bottaro, A. \& De Palma, P. $2010 b$ Optimal wave packets in a boundary layer and initial phases of a turbulent spot. J. Fluid Mech. 656, 231-259.

Chomaz, J. M. 2005 Global instabilities in spatially developing flows: non-normality and nonlinearity. Annu. Rev. Fluid Mech. 37, 357-392.

Corbett, P. \& Bottaro, A. 2000 Optimal perturbations for boundary layers subject to stream-wise pressure gradient. Phys. Fluids 12, 120-130.

Corino, E. R. \& BRodKey, R. S. 1969 A visual investigation of the wall region in turbulent flow. J. Fluid Mech. 37, 1.

Duguet, Y., BRAndt, L. \& LARSSON, B. R. J. 2010 Towards minimal perturbations in transitional plane Couette flow. Phys. Rev. E 82, 026316.

Duguet, Y., Schlatter, P. \& Henningson, D. 2009 Localized edge states in plane Couette flow. Phys. Fluids 21, 111701.

Eckhardt, B., Schneider, T. M., Hof, B. \& WesterweEl, J. 2007 Turbulence transition of pipe flow. Annu. Rev. Fluid Mech. 39, 447-468.

Faisst, H. \& Eckhardt, B. 2003 Travelling waves in pipe flow. Phys. Rev. Lett. 91, 224502.

FARrell, B. 1988 Optimal excitation of perturbations in viscous shear flow. Phys. Fluids 31, 2093-2102.

Gibson, J. F., Halcrow, J. \& Cvitanović, P. 2009 Equilibrium and travelling-wave solutions of plane Couette flow. J. Fluid Mech. 638, 243.

GuO, Y. \& Finlay, W. H. 1991 Splitting, merging and wave length selection of vortices in curved and/or rotating channel flow due to Eckhaus instability. J. Fluid Mech. 228, 661-691.

Hof, B., van Doorne, C. W. H., Westerweel, J., Nieuwstadt, F. T. M., Faisst, H., Eckhardt, B., Wedin, H., Kerswell, R. R. \& WAlefFe, F. 2004 Experimental observation of nonlinear traveling waves in turbulent pipe flow. Science 305, 1594-1598.

Hunt, J. C. R., Wray, A. \& Moin, P. 1988 Eddies, stream, and convergence zones in turbulent flows. Report CTR-S88, Center for Turbulence Research. 
JACobs, R. G. \& Durbin, P. A. 2001 Simulations of bypass transition. J. Fluid Mech. 428, $185-212$.

KAWAharA, G. \& KIDA, S. 2001 Periodic motion embedded in plane Couette turbulence: regeneration cycle and burst. J. Fluid Mech. 449, 291-300.

Kline, S. J., Reynold, W. C., Schraub, F. \& Rundstander, P. W. 1967 The structure of turbulent boundary layer flows. J. Fluid Mech. 30, 741-773.

LANDAHL, M. T. 1980 A note on an algebraic instability of inviscid parallel shear flows. J. Fluid Mech. 98, 243-251.

LuCHINI, P. 2000 Reynolds number indipendent instability of the Blasius boundary layer over a flat surface: optimal perturbations. J. Fluid Mech. 404, 289-309.

Marquet, O., Sipp, D., Chomaz, J.-M. \& Jacquin, L. 2008 Amplifier and resonator dynamics of a low-Reynolds-number recirculation bubble in a global framework. J. Fluid Mech. 605, 429-443.

Mellibovsky, F., Meseguer, A., Schneider, T. M. \& Eckhardt, B. 2009 Transition in localized pipe-flow turbulence. Phys. Rev. Lett. 103, 054502.

Monokrousos, A., Akervik, E., Brandt, L. \& Henningson, D. S. 2010 Global three-dimensional optimal disturbances in the Blasius boundary-layer flow using time steppers. J. Fluid Mech. 650, 181-214.

Monokrousos, A., Bottaro, A., Brandt, L., Di Vita, A. \& Henningson, D. S. 2011 Non-equilibrium thermodynamics and the optimal path to turbulence in shear flows. Phys. Rev. Lett. 106, 134502.

NAGAtA, M. 1990 Three-dimensional finite amplitude solutions in plane Couette flow. J. Fluid Mech. 217, 519-527.

PolAK, E. \& Ribière, G. 1969 Note sur la convergence de directions conjugées. Rev. Française Automat. Informat. Rech. Opér. 16, 35-43.

Pringle, C. C. T. \& Kerswell, R. R. 2010 Using nonlinear transient growth to construct the minimal seed for shear flow turbulence. Phys. Rev. Lett. 105, 154502.

Reddy, S. C., Schmid, P., Baggett, P. \& Henningson, D. S. 1998 On stability of streamwise streaks and transition thresholds in plane channel flows. J. Fluid Mech. 365, 269-303.

Robinson, S. K. 1991 Coherent motions in the turbulent boundary layer. Annu. Rev. Fluid Mech. 23, 601 .

SARIC, W. S., ReED, H. J. \& KeRSCHEN, E. 2002 Boundary-layer receptivity to free stream disturbances. Annu. Rev. Fluid Mech. 34, 291-319.

Schmid, P. \& Henningson, D. 2001 Stability and Transition in Shear Fows. Springer.

Schneider, T. M., Eckhardt, B. \& Yorke, J. A. 2007 Turbulence transition and the edge of chaos in pipe flow. Phys. Rev. Lett. 99, 034502.

Schneider, T. M., Gibson, J. F. \& Burke, J. 2010a Snakes and ladders: localized solutions of plane Couette flow. Phys. Rev. Lett. 104, 104501.

Schneider, T. M., MARINC, D. \& ECKhardT, B. $2010 \mathrm{~b}$ Localised edge states nucleate turbulence in extended plane Couette cells. J. Fluid Mech. 646, 441-451.

Schoppa, W. \& Huss Ain, F. 2002 Coherent structure generation in near-wall turbulence. J. Fluid Mech. 453, 57-108.

Singer, B. A. 1996 Characteristics of a young turbulent spot. Phys. Fluids 8, 509-512.

VERzICCO, R. \& ORLANDI, P. 1996 A finite-difference scheme for the three-dimensional incompressible flows in cylindrical coordinates. J. Comput. Phys. 123 (2), 402-414.

Viswanath, D. \& Cvitanovic, P. 2009 Stable manifolds and the transition to turbulence in pipe flow. J. Fluid Mech. 627, 215.

WALEFFE, F. 1995 Transition in shear flows: nonlinear normality versus non-normal linearity. Phys. Fluids 7, 3060-3066.

WALefFe, F. 1997 On a self-sustaining process in shear flows. Phys. Fluids 9, 883-900.

WalefFe, F. 1998 Three-dimensional states in plane shear flow. Phys. Rev. Lett. 81, 4140-4143.

WEDIN, H. \& KERSWELL, R. R. 2004 Exact coherent structures in pipe flow: travelling wave solutions. J. Fluid Mech. 508, 333-371.

Westin, K. J. A., Boiko, A. V., Klingmann, B. G. B., Kozlov, V. V. \& Alfredsson, P. H. 1994 Experiments in a boundary layer subject to free stream turbulence Part I: boundary layer structure and receptivity. J. Fluid Mech. 281, 193-218. 
Willmarth, W. W. \& LU, S. S. 1972 Structure of the Reynolds stress near the wall. J. Fluid Mech. 55, 65.

WU, X. \& MoIN, P. 2009 Direct numerical simulation of turbulence in a nominally-zero-pressuregradient flat-plate boundary layer. J. Fluid Mech. 630, 5-41.

Zuccher, S., Luchini, P. \& BotTARo, A. 2004 Algebraic growth in a blasius boundary layer: optimal and robust control by mean suction in the nonlinear regime. Eur. J. Mech. (B/Fluids) 513, 135-160. 